\title{
Quarkonium spectroscopy and perturbative QCD: Massive quark-loop effects
}

\author{
N. Brambilla \\ INFN and Dipartimento di Fisica dell'Università di Milano, via Celoria 16, 20133 Milan, Italy \\ Y. Sumino \\ Department of Physics, Tohoku University, Sendai, 980-8578, Japan
}

A. Vairo

Theory Division, CERN, 1211 Geneva 23, Switzerland

(Received 10 August 2001; published 7 January 2002)

\begin{abstract}
We study the spectra of the bottomonium and $B_{c}$ states within perturbative QCD up to order $\alpha_{\mathrm{s}}^{4}$. The $\mathcal{O}\left(\Lambda_{\mathrm{QCD}}\right)$ renormalon cancellation between the static potential and the pole mass is performed in the $\varepsilon$-expansion scheme. We extend our previous analysis by including the (dominant) effects of nonzero charmquark mass in loops up to the next-to-leading nonvanishing order $\varepsilon^{3}$. We fix the $b$-quark $\overline{\mathrm{MS}}$ mass $\bar{m}_{b}$ $\equiv m_{b}^{\overline{\mathrm{MS}}}\left(m_{b}^{\overline{\mathrm{MS}}}\right)$ on $\mathrm{Y}(1 S)$ and compute the higher levels. The effect of the charm mass decreases $\bar{m}_{b}$ by about 11 $\mathrm{MeV}$ and increases the $n=2$ and $n=3$ levels by about 70-100 MeV and 240-280 MeV, respectively. We provide an extensive quantitative analysis. The size of nonperturbative and higher order contributions is discussed by comparing the obtained predictions with the experimental data. An agreement of the perturbative predictions and the experimental data depends crucially on the precise value (inside the present error) of $\alpha_{\mathrm{s}}\left(M_{Z}\right)$. We obtain $m_{b}^{\overline{\mathrm{MS}}}\left(m_{b}^{\overline{\mathrm{MS}}}\right)=4190 \pm 20 \pm 25 \pm 3 \mathrm{MeV}$.
\end{abstract}

DOI: 10.1103/PhysRevD.65.034001

PACS number(s): $12.38 . \mathrm{Bx}, 14.40 . \mathrm{Nd}$

\section{INTRODUCTION}

Traditional methods for theoretical investigations of heavy-quarkonium spectra have been based on various phenomenological potential models in the frame of nonrelativistic quantum mechanics. These phenomenological potentials were tuned to reproduce the observed quarkonium spectra (and some other physical observables) and have been (in most cases) successful in predicting physical observables of the quarkonia, such as leptonic widths and transition rates among different levels, besides reproducing the energy levels. They have elucidated various properties of the bound states and essentially established, from consistency, the nonrelativistic nature of the quarks inside quarkonia. For some reviews we refer to [1]. An apparent deficit of these approaches is, however, the difficulty in relating the phenomenological parameters to the fundamental parameters of QCD.

Recently we have reported in [2] new results on the spectroscopy of heavy quarkonia (charmonium, bottomonium and $B_{c}$ ) computed in perturbative QCD up to order $\alpha_{\mathrm{s}}^{4}$, once the $\mathcal{O}\left(\Lambda_{\mathrm{QCD}}\right)$ renormalon cancellation between the static potential and the pole mass $[3,4]$ has been implemented. In order to realize this we have adopted the so-called $\varepsilon$-expansion scheme [5]. (In the present work we will use the same scheme.) The major results of that analysis have been as follows. (1) Once the cancellation of the leading renormalon has been incorporated, the perturbative series turns out to be convergent and to reproduce reasonably well the gross structure of the bottomonium spectrum at least up to some of the $n=3$ levels. (2) The constraints on nonperturbative and higher-order contributions to the bottomonium spectrum, set by the comparison of the calculation with the experimental data, indicate that these are smaller than usually believed.

In this paper we improve the analysis of [2] by including the effects of the non-zero charm-quark mass loops on the spectra of bottomonium and $B_{c}$. The typical scales of the bottomonium states as well as of the $B_{c}$ (as obtained in [2]) are either close to or less than the charm-quark mass. Hence, charm-mass effects are expected and actually turn out to be numerically not negligible. These effects have been computed for the relation between the pole-mass and the mass in the modified minimal subtraction scheme ( $\overline{\mathrm{MS}}$-mass) at leading (nonvanishing) order in [6] and at the next-to-leading order in the limit of small charm mass in [7]. In the bindingenergy expansion they have been calculated at leading (nonvanishing) order in [8] and at the next-to-leading order, only for the $Y(1 S)$ mass, in [7]. In the present work we implement and discuss the above results and derive some new formulas valid for the inclusion of charm-mass effects at the next-to-leading order for all quarkonium excited states. This will allow us to fully take into account charm-mass effects in the spectra of bottomonium and $B_{c}$ up to order $\varepsilon^{3}$ in the $\varepsilon$-expansion. Finally, we re-examine whether, by including these effects, the above conclusions (1) and (2) of [2] still hold.

The paper is organized as follows. In Sec. II we review the theoretical framework and the results of Ref. [2]. In Sec. III we present all the formulas needed for the inclusion of the nonzero charm-mass effects up to order $\varepsilon^{3}$ in the bottomonium and $B_{c}$ energy levels. In Sec. IV we perform the numerical analyses on the bottomonium and $B_{c}$ spectra that include the above effects. In Sec. V we give some conclusions. 


\section{QUARKONIUM SPECTRUM WITH MASSLESS QUARK LOOPS}

The heavy-quarkonium dynamics is characterized by at least two dynamically generated scales: the (soft) scale $m v$, of the order of the momentum transfer and the (ultrasoft) scale $m v^{2}$ of the order of the energy of the quark in the bound state ( $v$ being the typical size of the heavy-quark velocity in a quarkonium state). The magnitude and nature of the non-perturbative corrections depend on the relative size of $\Lambda_{\mathrm{QCD}}$ with respect to the dynamically generated scales. Different scenarios are then possible. These have been systematically studied in [9], where the effective field theories corresponding to them, generically denoted as potential nonrelativistic QCD [10], have been constructed. In [2] we have discussed our work in relation to these scenarios. That discussion applies also here. In order to facilitate the reading, let us summarize the main points. We assume to be in a situation where $m v \gg \Lambda_{\mathrm{QCD}}$ and the system to be mainly Coulombic. In this scenario nonperturbative corrections may be carried by local or nonlocal gluon condensates. Because of the fact that, in both cases, a direct numerical evaluation turns out to be quite uncertain, we chose not to correct explicitly our perturbative formulas with them (as done, for instance, in $[11,12])$, but rather to infer their size, as well as that of the neglected higher-order perturbative contributions from the eventual disagreement (and uncertainties) of the perturbative results from the experimental data. Among the neglected higher-order perturbative contributions we also include the $\alpha_{\mathrm{s}}^{5} \ln \alpha_{\mathrm{s}}$ contributions, which are known from [13], but whose dominance over other $\mathcal{O}\left(\alpha_{\mathrm{s}}^{5}\right)$ corrections is unclear. Therefore, using the effective field theory language of [9], we will consider in the following heavy quarkonium as described by potential nonrelativistic QCD in the perturbative regime up to order $\alpha_{\mathrm{s}}^{4}$. This will correspond to just considering the quarkonium spectrum up to that order as obtained from standard perturbative QCD.

\section{A. Perturbative expansions}

In this section, in order to set up some basic formulas, we review the energy (mass) of a quarkonium state $X$ made by two heavy quarks in the theory with $n_{l}$ massless quarks only. Namely, we do not take into account, for the moment, the effects of nonzero masses in the light-quark loops, neither in the binding energy nor in the pole-mass expansion. These will be considered in Sec. III.

We express the quarkonium mass as a series expansion in the $\overline{\mathrm{MS}}$ coupling constant $\alpha_{\mathrm{s}}^{\left(n_{l}\right)}(\mu)$ defined in the theory with $n_{l}$ massless quarks only. Consider the quantum-mechanical Hamiltonian

$$
H=m_{1, \text { pole }}+m_{2, \text { pole }}+H_{0}+H_{1}+H_{2}+\cdots
$$

where $^{1}$

$$
\begin{aligned}
H_{0}= & \frac{\vec{p}^{2}}{2 m_{r}}-\frac{4 \alpha_{\mathrm{s}}^{\left(n_{l}\right)}(\mu)}{3 r}, \\
H_{1}= & -\frac{4 \alpha_{\mathrm{s}}^{\left(n_{l}\right)}(\mu)}{3 r}\left(\frac{\alpha_{\mathrm{s}}^{\left(n_{l}\right)}(\mu)}{4 \pi}\right)\left\{2 \beta_{0}^{\left(n_{l}\right)} \ell+a_{1}^{\left(n_{l}\right)}\right\} \\
H_{2}= & -\frac{4 \alpha_{\mathrm{s}}^{\left(n_{l}\right)}(\mu)}{3 r}\left(\frac{\alpha_{\mathrm{s}}^{\left(n_{l}\right)}(\mu)}{4 \pi}\right)^{2}\left\{\beta_{0}^{\left(n_{l}\right) 2}\left(4 \ell^{2}+\frac{\pi^{2}}{3}\right)+2\left(\beta_{1}^{\left(n_{l}\right)}+2 \beta_{0}^{\left(n_{l}\right)} a_{1}^{\left(n_{l}\right)}\right) \ell+a_{2}^{\left(n_{l}\right)}\right\}-\frac{(1+3 x) \vec{p}^{4}}{32 m_{r}^{3}}+\frac{\pi(1+x) \alpha_{\mathrm{s}}^{\left(n_{l}\right)}(\mu)}{3 m_{r}^{2}} \delta^{3}(\vec{r}) \\
& \left.-\frac{(1-x) \alpha_{\mathrm{s}}^{\left(n_{l}\right)}(\mu)}{6 m_{r}^{2} r}(\vec{p})^{2}+\frac{1}{r^{2}} r_{i} r_{j} p_{j} p_{i}\right)-\frac{(1-x) \alpha_{\mathrm{s}}^{\left(n_{l}\right)}(\mu)}{6 m_{r}^{2}}\left\{\frac{S^{2}}{r^{3}}-3 \frac{(\vec{S} \cdot \vec{r})^{2}}{r^{5}}-\frac{4 \pi}{3}\left(2 S^{2}-3\right) \delta^{3}(\vec{r})\right\}-\frac{\alpha_{\mathrm{s}}^{\left(n_{l}\right)}(\mu)^{2}}{m_{r} r^{2}} \\
& +\frac{(3-x) \alpha_{\mathrm{s}}^{\left(n_{l}\right)}(\mu)}{6 m_{r}^{2} r^{3}} \vec{L} \cdot \vec{S}+\frac{\alpha_{\mathrm{s}}^{\left(n_{l}\right)}(\mu)}{3 r^{3}}\left(\frac{1}{m_{1, \mathrm{pole}}^{2}}-\frac{1}{m_{2, \mathrm{pole}}^{2}}\right) \vec{L} \cdot\left(\vec{S}_{1}-\vec{S}_{2}\right) .
\end{aligned}
$$

We have defined the reduced pole mass as $m_{r}=m_{1, \text { pole }} m_{2, \text { pole }} /\left(m_{1, \text { pole }}+m_{2, \text { pole }}\right) \quad$ and $\quad x=1-4 m_{r} /$ $\left(m_{1, \text { pole }}+m_{2 \text {,pole }}\right)$; when the two masses are equal, $x=0$. The $\beta_{k}^{\left(n_{l}\right)}$ 's denote the coefficients of the QCD beta function:

$$
\beta_{0}^{\left(n_{l}\right)}=11-\frac{2}{3} n_{l}, \quad \beta_{1}^{\left(n_{l}\right)}=102-\frac{38}{3} n_{l},
$$

\section{$\ell, a_{1}^{\left(n_{l}\right)}$ and $a_{2}^{\left(n_{l}\right)}$ are given by}

\footnotetext{
${ }^{1}$ The term $\mathrm{H}_{2}$, in its unitary-equivalent form, can be read from Ref. [11] supplemented with the two-loop static potential calculated in [14].
} 


$$
\begin{aligned}
\ell= & \ln (\mu r)+\gamma_{E}, \\
a_{1}^{\left(n_{l}\right)}= & \frac{31}{3}-\frac{10}{9} n_{l}, \\
a_{2}^{\left(n_{l}\right)}= & \frac{4343}{18}+36 \pi^{2}+66 \zeta_{3}-\frac{9 \pi^{4}}{4} \\
& -\left(\frac{1229}{27}+\frac{52 \zeta_{3}}{3}\right) n_{l}+\frac{100}{81} n_{l}^{2} .
\end{aligned}
$$

Up to $\mathcal{O}\left(\alpha_{\mathrm{s}}^{4} m\right)$, the energy of a heavy quarkonium state $X$, identified by the quantum numbers $n, l, s$ and $j$, is derived from the perturbative expansions of the energy eigenvalues ${ }^{2}$ of the above Hamiltonian:

$$
\begin{aligned}
E_{X}\left(\mu, \alpha_{\mathrm{s}}^{\left(n_{l}\right)}(\mu), m_{i, \text { pole }}\right)= & m_{1, \text { pole }}+m_{2, \text { pole }} \\
& +E_{\text {bin }, X}^{n_{l}}\left(\mu, \alpha_{\mathrm{s}}^{\left(n_{l}\right)}(\mu), m_{i, \text { pole }}\right) \\
E_{\mathrm{bin}, X}^{n_{l}}\left(\mu, \alpha_{\mathrm{s}}^{\left(n_{l}\right)}(\mu), m_{i, \text { pole }}\right)= & -\frac{8}{9 n^{2}} \alpha_{\mathrm{s}}^{\left(n_{l}\right)}(\mu)^{2} m_{r} \\
& \times \sum_{k=0}^{2} \varepsilon^{k+1}\left(\frac{\alpha_{\mathrm{s}}^{\left(n_{l}\right)}(\mu)}{\pi}\right)^{k} P_{k}\left(L_{n l}\right),
\end{aligned}
$$

where $\varepsilon=1$ is the parameter that will be used in order to properly organize the perturbative expansion in view of the $\mathcal{O}\left(\Lambda_{\mathrm{QCD}}\right)$ renormalon cancellation [5]. $P_{k}\left(L_{n l}\right)$ is a $k^{\text {th }}$-degree polynomial of $L_{n l} \equiv \ln \left[3 n \mu /\left(8 \alpha_{\mathrm{s}}^{\left(n_{l}\right)}(\mu) m_{r}\right)\right]$ $+S_{1}(n+l)+\frac{5}{6}$, and the harmonic sums are defined as $S_{p}(q) \equiv \sum_{k=1}^{q}\left(1 / k^{p}\right)$. It is convenient to decompose the polynomials into renormalization-group invariant subsets:

$$
\begin{aligned}
P_{0}= & 1 \\
P_{1}= & \beta_{0}^{\left(n_{l}\right)} L_{n l}+c_{1} \\
P_{2}= & \frac{3}{4} \beta_{0}^{\left(n_{l}\right) 2} L_{n l}^{2}+\left(-\frac{1}{2} \beta_{0}^{\left(n_{l}\right) 2}+\frac{1}{4} \beta_{1}^{\left(n_{l}\right)}+\frac{3}{2} \beta_{0}^{\left(n_{l}\right)} c_{1}\right) L_{n l} \\
& +c_{2}^{\left(n_{l}\right)} .
\end{aligned}
$$

$c_{1}$ and $c_{2}^{\left(n_{l}\right)}$ are given $\mathrm{by}^{3}$

\footnotetext{
${ }^{2}$ The full formula up to $\mathcal{O}\left(\alpha_{\mathrm{s}}^{4} m\right)$ for the $S$ state spectrum was derived in [12] and later confirmed in [15]; additional corrections necessary for the spectrum of $l \geqslant 1$ states can be found in [11] and the formula for the unequal mass and $l=0$ case in [16].

${ }^{3}$ This corrects the formula given for $c_{2}^{\left(n_{l}\right)}$ in [2] in the situation when $m_{1 \text {,pole }} \neq m_{2 \text {,pole }}$ and $l>0$ hold at the same time. Since, however, all the numerical analyses of [2] correspond to the equal-mass case or to $l=0$ states, they remain valid.
}

$$
\begin{aligned}
c_{1}= & -4 \\
c_{2}^{\left(n_{l}\right)}= & -\frac{16 \pi^{2}\{2 s(s+1)(1-x)+3 x\}}{27 n} \delta_{l 0} \\
& +\frac{8 \pi^{2} \lambda(l, s, j)}{9 n l(l+1)(2 l+1)}\left(1-\delta_{l 0}\right)+\beta_{0}^{\left(n_{l}\right) 2} \nu(n, l) \\
& -\frac{(11+x) \pi^{2}}{9 n^{2}}+\frac{68 \pi^{2}}{9 n(2 l+1)}+\frac{473}{16}+\frac{9 \pi^{2}}{2}+\frac{33 \zeta_{3}}{4} \\
& -\frac{9 \pi^{4}}{32}-n_{l}\left(\frac{109}{72}+\frac{13 \zeta_{3}}{6}\right)
\end{aligned}
$$

where $\lambda(l, s, j)$ represents the fine and hyperfine splittings for $l>0$. If $j=l \pm 1$ or $m_{1, \text { pole }}=m_{2, \text { pole }}(x=0)$, it is given by

$$
\lambda(l, s, j)=-(1-x) D_{S}-(3-x) X_{L S},
$$

with

$$
D_{S} \equiv\left\langle 3 \frac{(\vec{r} \cdot \vec{S})^{2}}{r^{2}}-\vec{S}^{2}\right\rangle=\frac{2 l(l+1) s(s+1)-3 X_{L S}-6 X_{L S}^{2}}{(2 l-1)(2 l+3)}
$$

$X_{L S} \equiv\langle\vec{L} \cdot \vec{S}\rangle=\frac{1}{2}[j(j+1)-l(l+1)-s(s+1)]$

If $j=l$ and $m_{1, \text { pole }} \neq m_{2, \text { pole }}(x>0)$, the last term of Eq. (4) induces a mixing between the $s=0$ and $s=1$ states, and the total spin $s$ is no longer a good quantum number (see, for instance, [17]). In this case, the splitting is given by ${ }^{4}$

$$
\lambda(l, \pm, j=l)=1 \pm \sqrt{1+4 x l(l+1)} .
$$

The term $\nu(n, l)$ is given $\mathrm{by}^{5}$

$$
\begin{aligned}
\nu(n, l)= & \frac{\pi^{2}}{8}-\frac{1}{2} S_{2}(n+l) \\
& +\frac{n}{2} \frac{(n+l) !}{(n-l-1) !} \sum_{k=1}^{\infty} \frac{(n-l+k-1) !}{(n+l+k) ! k^{3}} \\
& +\frac{(n-l-1) !}{2(n+l) !} \sum_{k=1}^{n-l-1} \frac{(2 l+k) !(2 k+2 l-n)}{(k-1) !(k+l-n)^{3}} .
\end{aligned}
$$

It is understood that the last term is zero if $n-l<2$.

Next we rewrite the series expansion of $E_{X}$ in terms of the $\overline{\mathrm{MS}}$ masses. This is done by expressing the pole masses $m_{i, \text { pole }}$ in terms of the renormalization-group-invariant $\overline{\mathrm{MS}}$

${ }^{4}$ The corresponding energy eigenstate reads $| \pm\rangle=[b|s=0\rangle$ $\left.-\lambda_{ \pm}|s=1\rangle\right] / \sqrt{|b|^{2}+\lambda_{ \pm}^{2}}$, where $b=2 \sqrt{x l(l+1)}$ up to a convention-dependent phase.

${ }^{5}$ The infinite sum can easily be evaluated analytically in terms of $\zeta_{3}$, etc. for given values of $n$ and $l$, e.g. by using MATHEMATICA. 
masses $\bar{m}_{i} \equiv m_{i, \overline{\mathrm{MS}}}\left(m_{i, \overline{\mathrm{MS}}}\right)$. In the theory with $n_{l}$ massless quarks only, we have up to $\mathcal{O}\left(\alpha_{\mathrm{s}}^{3}\right)$ :

$$
\begin{aligned}
m_{i, \text { pole }}= & \bar{m}_{i}\left\{1+\frac{4}{3} \varepsilon \frac{\alpha_{\mathrm{s}}^{\left(n_{l}\right)}\left(\bar{m}_{i}\right)}{\pi}+\varepsilon^{2}\left(\frac{\alpha_{\mathrm{s}}^{\left(n_{l}\right)}\left(\bar{m}_{i}\right)}{\pi}\right)^{2} d_{1}^{\left(n_{l}\right)}\right. \\
& \left.+\varepsilon^{3}\left(\frac{\alpha_{\mathrm{s}}^{\left(n_{l}\right)}\left(\bar{m}_{i}\right)}{\pi}\right)^{3} d_{2}^{\left(n_{l}\right)}\right\} .
\end{aligned}
$$

The coefficients $d_{1}^{\left(n_{l}\right)}$ and $d_{2}^{\left(n_{l}\right)}$ are given in Appendix A.

Note that the counting in $\varepsilon$ in Eq. (10) and Eq. (21) does not reflect the order in $\alpha_{\mathrm{s}}$ but the wanted renormalon cancellation [5]. One way to understand this is to consider that in the sum of the pole quark masses and the static QCD potential, $\Sigma_{i} m_{i \text {,pole }}+V_{\mathrm{QCD}}(r)$, the renormalon cancellation takes place without reordering of power counting in $\alpha_{\mathrm{s}}[3,4]$. The extra power of $\alpha_{\mathrm{s}}$ comes in the energy-level expansion when the dynamical variable $r^{-1}$ is replaced by the dynamical scale $\left\langle n l s j\left|r^{-1}\right| n l s j\right\rangle \sim C_{F} \alpha_{\mathrm{s}} m_{r} / n$, where $C_{F}=4 / 3$. More- over, in order to realize the renormalon cancellation at each order of the expansion, it is necessary to expand $m_{i \text {,pole }}$ and $E_{\text {bin } X}^{n_{l}}$ in the same coupling [18,5,19]; we therefore express $\alpha_{\mathrm{s}}^{\left(n_{l}\right)}\left(\bar{m}_{i}\right)$ in Eq. (21) in terms of $\alpha_{\mathrm{s}}^{\left(n_{l}\right)}(\mu)$ :

$$
\begin{aligned}
\alpha_{\mathrm{s}}^{\left(n_{l}\right)}\left(\bar{m}_{i}\right)= & \alpha_{\mathrm{s}}^{\left(n_{l}\right)}(\mu)\left\{1+\varepsilon \frac{\alpha_{\mathrm{s}}^{\left(n_{l}\right)}(\mu)}{\pi} \frac{\beta_{0}^{\left(n_{l}\right)}}{2} \ln \left(\frac{\mu}{\bar{m}_{i}}\right)\right. \\
& +\varepsilon^{2}\left(\frac{\alpha_{\mathrm{s}}^{\left(n_{l}\right)}(\mu)}{\pi}\right)^{2}\left[\frac{\beta_{0}^{\left(n_{l}\right) 2}}{4} \ln ^{2}\left(\frac{\mu}{\bar{m}_{i}}\right)\right. \\
& \left.\left.+\frac{\beta_{1}^{\left(n_{l}\right)}}{8} \ln \left(\frac{\mu}{\bar{m}_{i}}\right)\right]\right\} .
\end{aligned}
$$

Inserting Eqs. (22) and (21) into Eqs. (10) and (9), we get an expression for the energy levels of the heavy quarkonium states, which depends on $\mu, \alpha_{\mathrm{s}}^{\left(n_{l}\right)}(\mu)$ and $\bar{m}_{i}$, that we can organize as an expansion in $\varepsilon$ up to order $\varepsilon^{3}$ :

$$
\begin{aligned}
E_{X}\left(\mu, \alpha_{\mathrm{s}}^{\left(n_{l}\right)}(\mu), \bar{m}_{i}\right)= & {\left[\sum_{i=1}^{2} \bar{m}_{i}\left\{1+\frac{4}{3} \varepsilon \frac{\alpha_{\mathrm{s}}^{\left(n_{l}\right)}\left(\bar{m}_{i}\right)}{\pi}+\varepsilon^{2}\left(\frac{\alpha_{\mathrm{s}}^{\left(n_{l}\right)}\left(\bar{m}_{i}\right)}{\pi}\right)^{2} d_{1}^{\left(n_{l}\right)}+\varepsilon^{3}\left(\frac{\alpha_{\mathrm{s}}^{\left(n_{l}\right)}\left(\bar{m}_{i}\right)}{\pi}\right)^{3} d_{2}^{\left(n_{l}\right)}\right\}\right.} \\
& \left.+E_{\text {bin }, X}^{n_{l}}\left(\mu, \alpha_{\mathrm{s}}^{\left(n_{l}\right)}(\mu), \bar{m}_{i}\left(1+\frac{4}{3} \varepsilon \frac{\alpha_{\mathrm{s}}^{\left(n_{l}\right)}\left(\bar{m}_{i}\right)}{\pi}+\varepsilon^{2}\left(\frac{\alpha_{\mathrm{s}}^{\left(n_{l}\right)}\left(\bar{m}_{i}\right)}{\pi}\right)^{2} d_{1}^{\left(n_{l}\right)}\right\}\right)\right]_{\alpha_{\mathrm{s}}\left(\bar{m}_{i}\right)=\text { Eq. (22) }} \\
\equiv & \bar{m}_{1}+\bar{m}_{2}+E_{X}^{n_{l}(1)}\left(\mu, \alpha_{\mathrm{s}}^{\left(n_{l}\right)}(\mu), \bar{m}_{i}\right) \varepsilon+E_{X}^{n_{l}(2)}\left(\mu, \alpha_{\mathrm{s}}^{\left(n_{l}\right)}(\mu), \bar{m}_{i}\right) \varepsilon^{2}+E_{X}^{n_{l}(3)}\left(\mu, \alpha_{\mathrm{s}}^{\left(n_{l}\right)}(\mu), \bar{m}_{i}\right) \varepsilon^{3} \\
& +\cdots .
\end{aligned}
$$

Since the counting in $\varepsilon$ explicitly realizes the order $\Lambda_{\mathrm{QCD}}$ renormalon cancellation, and since $\alpha_{\mathrm{s}}$ and $\bar{m}_{i}$ are short-range quantities, the obtained perturbative expansion (24) is expected to show a better convergence than Eq. (10).

\section{B. Physical parameters}

The input value for $\alpha_{\mathrm{s}}$ that we will use is [20]

$$
\alpha_{\mathrm{s}}^{(5)}\left(M_{Z}\right)=0.1181 \pm 0.0020 \text {. }
$$

Throughout this paper, we evolve the coupling and match it to the couplings of the theory with $n_{l}=4$ and 3 successively, by solving the renormalization-group equation perturbatively (analytically) at 4 loops [Eqs. (3) and (11) of Ref. [21]]. ${ }^{6} \mathrm{We}$ obtain

$$
\Lambda \frac{(5)}{\mathrm{MS}}=210_{-23}^{+24} \mathrm{MeV}
$$

\footnotetext{
${ }^{6}$ We take the matching scales as $\bar{m}_{b}$ and $\bar{m}_{c}$, respectively.
}

$$
\begin{aligned}
& \Lambda \frac{(4)}{\mathrm{MS}}=292_{-28}^{+30} \mathrm{MeV}, \\
& \Lambda \frac{(3)}{\mathrm{MS}}=333_{-28}^{+31} \mathrm{MeV} .
\end{aligned}
$$

In Ref. [2] we calculated the bottomonium spectrum in the $m_{c}=0$ case, i.e. directly from the above Eq. (24). ${ }^{7}$ In particular, by fitting the theoretical values of the masses of the $\Upsilon(1 S)$ and $J / \psi$ with the experimental ones, $\bar{m}_{b}$ and $\bar{m}_{c}$ were found to be

$$
\begin{aligned}
& \bar{m}_{b}=4201_{+18}^{-19} \mathrm{MeV} \quad \text { (without charm-mass effects), } \\
& \bar{m}_{c}=1237_{+16}^{-16} \mathrm{MeV},
\end{aligned}
$$

${ }^{7}$ In Ref. [2] major numerical results have been presented using the coupling $\alpha_{\mathrm{s}}^{\left(n_{l}\right)}(\mu)$ obtained by solving the 4-loop renormalizationgroup equation numerically. Here, we present the results computed using the above analytic coupling [corresponding to Table 2 column (i) of [2]]. 
a)

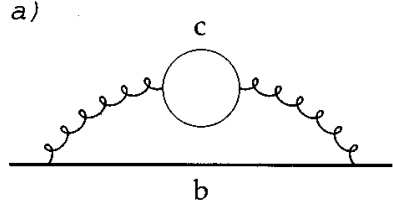

b)

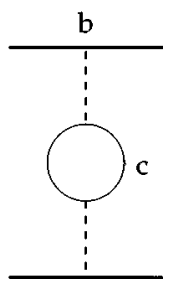

FIG. 1. Feynman graphs responsible for the leading charm-mass effects to the pole mass (a) and to the Coulomb potential (b).

where we quote only the errors due to the uncertainty in $\alpha_{\mathrm{s}}^{(5)}\left(M_{Z}\right)$ given in Eq. (25). We refer to [2] for an extended analysis of the other sources of uncertainty in the above determination. In [2], with these values of the quark masses as input, other bottomonium masses have been calculated. The masses of the states $2 S_{1}, 1 P_{0}, 1 P_{1}, 1 P_{2}, 2 P_{0}$ and $3 S_{1}$ could be determined in a reliable way in the sense of [2] (see also Sec. IV).

\section{CHARM-MASS EFFECTS}

In this section we discuss how Eqs. (9) and (21) get modified in the bottomonium case by including finite charm-mass effects up to order $\varepsilon^{3}$. These effects are generated by charm loop insertions and start at $\mathcal{O}\left(\alpha_{\mathrm{s}}^{3}\right)$ in the first case [Fig. 1(b)] and at $\mathcal{O}\left(\alpha_{\mathrm{s}}^{2}\right)$ in the latter case [Fig. 1(a)]. Therefore, considering a charm with a nonzero mass modifies the righthand side of Eq. (9) (taken in the bottomonium case and with $n_{l}=4$ active and massless flavors) and of Eq. (21) (taken in the bottom case and with $n_{l}=4$ active and massless flavors) respectively by an amount

$$
\begin{aligned}
\left(\delta E_{b \bar{b}}\right)_{m_{c}} & =\varepsilon^{2}\left(\delta E_{b \bar{b}}\right)_{m_{c}}^{(1)}+\varepsilon^{3}\left(\delta E_{b \bar{b}}\right)_{m_{c}}^{(2)}, \\
\left(\delta m_{b}\right)_{m_{c}} & =\varepsilon^{2}\left(\delta m_{b}\right)_{m_{c}}^{(1)}+\varepsilon^{3}\left(\delta m_{b}\right)_{m_{c}}^{(2)} .
\end{aligned}
$$

The term $\left(\delta E_{b \bar{b}}\right)_{m}^{(1)}$ has been calculated for all quantum numbers in [8]; $\left(\delta m_{b}\right)_{m_{c}}^{(1)}$ has been calculated in [6]; $\left(\delta E_{b \bar{b}}\right)_{m_{c}}^{(2)}$ has been calculated in [7] for the $1 S$ bottomonium level, but is unknown for higher quantum numbers; $\left(\delta m_{b}\right)_{m}^{(2)}$ has been calculated in [7] in the limit of $m_{c} \rightarrow 0$, keeping only the (leading) linear contribution in the charm mass ("linear approximation").

In the following we will analyze these different contributions. Our main result will be the calculation of the dominant contribution to $\left(\delta E_{b \bar{b}}\right)_{m_{c}}^{(2)}$ (at the level of 95\%) for all quantum numbers. Hence, we will be in a position to account for the finite charm-mass effects in the bottomonium spectrum up to order $\varepsilon^{3}$.

\section{A. Order- $\varepsilon^{2}$ effects}

The $\mathcal{O}\left(\varepsilon^{2}\right)$ effects in Eq. (31) and Eq. (32) stem from the graphs (b) and (a), respectively, of Fig. 1. In [6] it was found that

$$
\begin{aligned}
\left(\delta m_{b}\right)_{m_{c}}^{(1)}= & \frac{\bar{m}_{b}}{3}\left(\frac{\alpha_{\mathrm{s}}^{(4)}\left(\bar{m}_{b}\right)}{\pi}\right)^{2}\left[\ln ^{2}(\xi)+\frac{\pi^{2}}{6}-\left(\ln (\xi)+\frac{3}{2}\right) \xi^{2}\right. \\
& +(1+\xi)\left(1+\xi^{3}\right)\left(\operatorname{Li}_{2}(-\xi)-\frac{1}{2} \ln ^{2}(\xi)\right. \\
& \left.+\ln (\xi) \ln (1+\xi)+\frac{\pi^{2}}{6}\right)+(1-\xi)\left(1-\xi^{3}\right) \\
& \left.\times\left(\operatorname{Li}_{2}(\xi)-\frac{1}{2} \ln ^{2}(\xi)+\ln (\xi) \ln (1-\xi)-\frac{\pi^{2}}{3}\right)\right]
\end{aligned}
$$

where $\xi=\bar{m}_{c} / \bar{m}_{b}$. It is useful to define

$$
\begin{aligned}
\left(\delta m_{b}\right)_{m_{c} \rightarrow 0}^{(1)}= & \frac{\left(\alpha_{\mathrm{s}}^{(4)}\left(\bar{m}_{b}\right)\right)^{2}}{6} \bar{m}_{c}, \\
\left(\delta m_{b}\right)_{m_{c} \rightarrow \infty}^{(1)}= & \frac{\bar{m}_{b}}{3}\left(\frac{\alpha_{\mathrm{s}}^{(4)}\left(\bar{m}_{b}\right)}{\pi}\right)^{2}\left[\frac{151}{72}+\frac{\pi^{2}}{6}\right. \\
& \left.+\frac{13}{6} \ln \left(\frac{\bar{m}_{c}}{\bar{m}_{b}}\right)+\ln ^{2}\left(\frac{\bar{m}_{c}}{\bar{m}_{b}}\right)\right],
\end{aligned}
$$

where $\left(\delta m_{b}\right)_{m_{c} \rightarrow 0}^{(1)}$ corresponds to the expansion of $\left(\delta m_{b}\right)_{m_{c}}^{(1)}$ for $m_{c} \rightarrow 0$ [up to $\mathcal{O}\left(m_{c}^{2}\right)$ corrections] and $\left(\delta m_{b}\right)_{m_{c} \rightarrow \infty}^{(1)}$ corresponds to the expansion of $\left(\delta m_{b}\right)_{m_{c}}^{(1)}$ for $m_{c} \rightarrow \infty$ [up to $\mathcal{O}\left(1 / m_{c}^{2}\right)$ corrections]. In the first plot of Fig. 2 we show $\left(\delta m_{b}\right)_{m_{c}}^{(1)}$ as a function of $\bar{m}_{c}$ for $\bar{m}_{b}=4.201 \mathrm{GeV}$ [the value is taken from Eq. (29)]. Here and in the following, if not specified differently, $\alpha_{\mathrm{s}}^{\left(n_{l}\right)}$ is calculated from $\alpha_{\mathrm{s}}^{(5)}\left(M_{Z}\right)$ $=0.1181$ [central values of Eqs. (26)-(28)]. In the region of interest, $1 \mathrm{GeV} \lesssim \bar{m}_{c} \lesssim 1.5 \mathrm{GeV},\left(\delta m_{b}\right)_{m_{c}}^{(1)}$ turns out to be approximated by $\left(\delta m_{b}\right)_{m_{c} \rightarrow 0}^{(1)}$ reasonably well, while $\left(\delta m_{b}\right)_{m_{c} \rightarrow \infty}^{(1)}$ is far off. More specifically at the value $\bar{m}_{c}$ $=1.237 \mathrm{GeV}(\xi=0.294)$, taken from Eq. (30), we have

$$
\left(\delta m_{b}\right)_{m_{c}}^{(1)} \simeq 9.1 \mathrm{MeV}, \quad\left(\delta m_{b}\right)_{m_{c} \rightarrow 0}^{(1)} \simeq 10.5 \mathrm{MeV},
$$

$$
\left(\delta m_{b}\right)_{m_{c} \rightarrow \infty}^{(1)} \simeq 18.7 \mathrm{MeV} .
$$

The error of the "linear approximation" is about $15 \%$, which agrees with the analysis done in [7].

Since, in order to realize the renormalon cancellation, the coupling constant $\alpha_{\mathrm{s}}^{(4)}\left(\bar{m}_{b}\right)$ has to be expanded around the scale $\mu$, in the second plot of Fig. 2 we show $\left(\delta m_{b}\right)_{m_{c}}^{(1)}$, $\left(\delta m_{b}\right)_{m_{c} \rightarrow 0}^{(1)}$ and $\left(\delta m_{b}\right)_{m_{c} \rightarrow \infty}^{(1)}$ as functions of $\mu$ at $\bar{m}_{c}$ $=1.237 \mathrm{GeV}$ and $\bar{m}_{b}=4.201 \mathrm{GeV}$, when $\alpha_{\mathrm{s}}^{(4)}\left(\bar{m}_{b}\right)$ is substituted by $\alpha_{\mathrm{s}}^{(4)}(\mu)$ in Eqs. (33)-(35). Also this plot confirms that $\left(\delta m_{b}\right)_{m_{c} \rightarrow 0}^{(1)}$ approximates $\left(\delta m_{b}\right)_{m_{c}}^{(1)}$ reasonably well, while $\left(\delta m_{b}\right)_{m_{c} \rightarrow \infty}^{(1)}$ is far off.

In [8] it was found that 

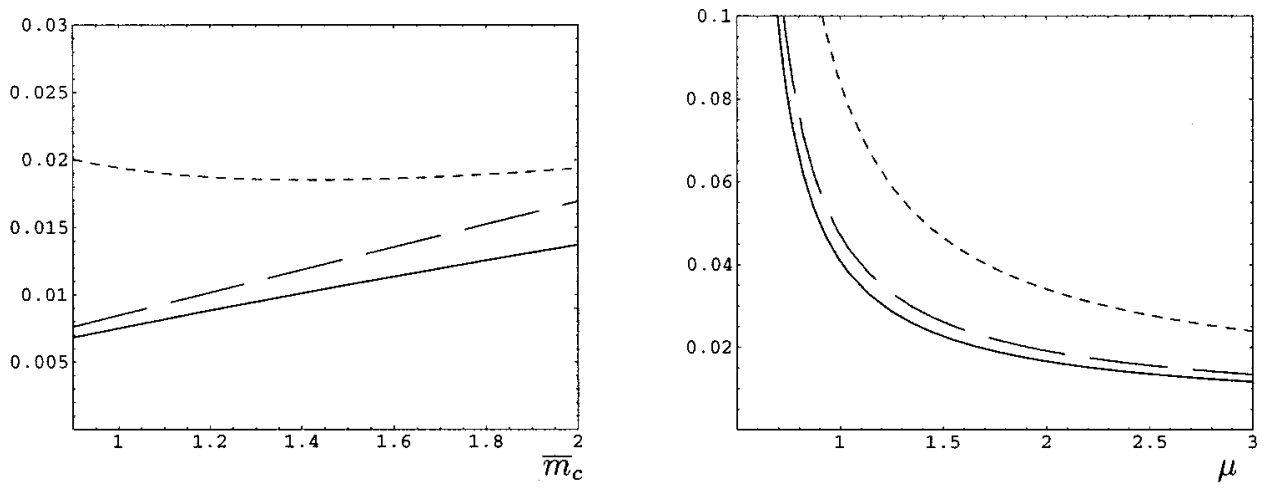

FIG. 2. The first figure shows $\left(\delta m_{b}\right)_{m_{c}}^{(1)}$ (continuous line), $\left(\delta m_{b}\right)_{m_{c} \rightarrow 0}^{(1)}$ (dashed line), $\left(\delta m_{b}\right)_{m_{c} \rightarrow \infty}^{(1)}$ (dotted line), as a function of $\bar{m}_{c}$ for $\bar{m}_{b}=4.201 \mathrm{GeV}$. The second figure shows $\left(\delta m_{b}\right)_{m_{c}}^{(1)}$ (continuous line), $\left(\delta m_{b}\right)_{m_{c} \rightarrow 0}^{(1)}$ (dashed line), $\left(\delta m_{b}\right)_{m_{c} \rightarrow \infty}^{(1)}($ dotted line), as a function of $\mu$, when $\alpha_{\mathrm{s}}^{(4)}\left(\bar{m}_{b}\right)$ is replaced by $\alpha_{\mathrm{s}}^{(4)}(\mu)$, for $\bar{m}_{b}=4.201 \mathrm{GeV}$ and $\bar{m}_{c}=1.237 \mathrm{GeV}$. The units are GeV.

$$
\begin{aligned}
\left(\delta E_{b \bar{b}}\right)_{m_{c}}^{(1)}= & \frac{\bar{m}_{b}\left(C_{F} \alpha_{\mathrm{s}}^{(4)}(\mu)\right)^{2}}{4 n^{2}} \frac{\alpha_{\mathrm{s}}^{(4)}(\mu)}{3 \pi}\left\{-\frac{3 \pi}{2} n \bar{\rho}+(n(2 n+1)+(n+l)(n-l-1)) \bar{\rho}^{2}-\pi n\left(\frac{1}{3}(n+1)(2 n+1)\right.\right. \\
& +(n+l)(n-l-1)) \bar{\rho}^{3}+2 \ln \left(\begin{array}{c}
2 \\
\bar{\rho}
\end{array}\right)-2(\psi(n+l+1)-\psi(1))-\frac{2}{(2 n-1) !} \sum_{k=0}^{n-l-1}\left(\begin{array}{c}
n-l-1 \\
k
\end{array}\right) \\
& \left.\left.\times\left(\begin{array}{c}
n+l \\
2 l+1+k
\end{array}\right) \bar{\rho}^{2(n-l-1-k)} \frac{d^{2 n-1}}{d \bar{\rho}^{2 n-1}}\left[-\frac{\sqrt{\bar{\rho}-1}}{\rho^{2 k+2 l+1}}\right)\right]\right\}
\end{aligned}
$$

where $\bar{\rho}=2 n \bar{m}_{c} /\left(\bar{m}_{b} C_{F} \alpha_{\mathrm{s}}^{(4)}(\mu)\right)$. Again, it is useful to define

$$
\begin{aligned}
\left(\delta E_{b \bar{b}}\right)_{m_{c} \rightarrow 0}^{(1)}= & -\frac{C_{F}\left(\alpha_{\mathrm{s}}^{(4)}(\mu)\right)^{2}}{4} \bar{m}_{c}, \\
\left(\delta E_{b \bar{b}}\right)_{m_{c} \rightarrow \infty}^{(1)}= & \frac{\bar{m}_{b}\left(C_{F} \alpha_{\mathrm{s}}^{(4)}(\mu)\right)^{2}}{4 n^{2}} \frac{\alpha_{\mathrm{s}}^{(4)}(\mu)}{3 \pi} \\
& \times\left\{2 \ln \left(\frac{2}{\bar{\rho}}\right)-\frac{5}{3}-2(\psi(n+l+1)-\psi(1))\right\},
\end{aligned}
$$

where $\left(\delta E_{b \bar{b}}\right)_{m_{c} \rightarrow 0}^{(1)}$ corresponds to the expansion of $\left(\delta E_{b \bar{b}}\right)_{m}^{(1)}$ for $m_{c} \rightarrow 0$ [up to $\mathcal{O}\left(m_{c}^{2}\right)$ corrections $]$ and $\left(\delta E_{b \bar{b}}\right)_{m_{c} \rightarrow \infty}^{(1)}$ corresponds to the expansion of $\left(\delta E_{b \bar{b}}\right)_{m_{c}}^{(1)}$ for $m_{c} \rightarrow \infty$ [up to $\mathcal{O}\left(1 / m_{c}^{2 l+2}\right)$ corrections]. In Fig. 3 we show $\left(\delta E_{b \bar{b}}\right)_{m_{c}}^{(1)}$ as a function of $\bar{m}_{c}$ for $\bar{m}_{b}=4.201 \mathrm{GeV}$. We plot the functions (from up to down) corresponding to the $1 S$ bottomonium level $(\mu=2.446 \mathrm{GeV})$, the $2 S$ bottomonium level $(\mu=1.065 \mathrm{GeV})$, and the $3 S$ bottomonium level $(\mu$ $=0.724 \mathrm{GeV})$. The scales $\mu$ correspond to the extremum of $E_{b \bar{b}}$ [defined in Eq. (23) $]$ at $\bar{m}_{b}=4.201 \mathrm{GeV}$. It is noteworthy that $\left(\delta E_{b \bar{b}}\right)_{m_{c} \rightarrow 0}^{(1)}$ does not depend explicitly on the quarko-

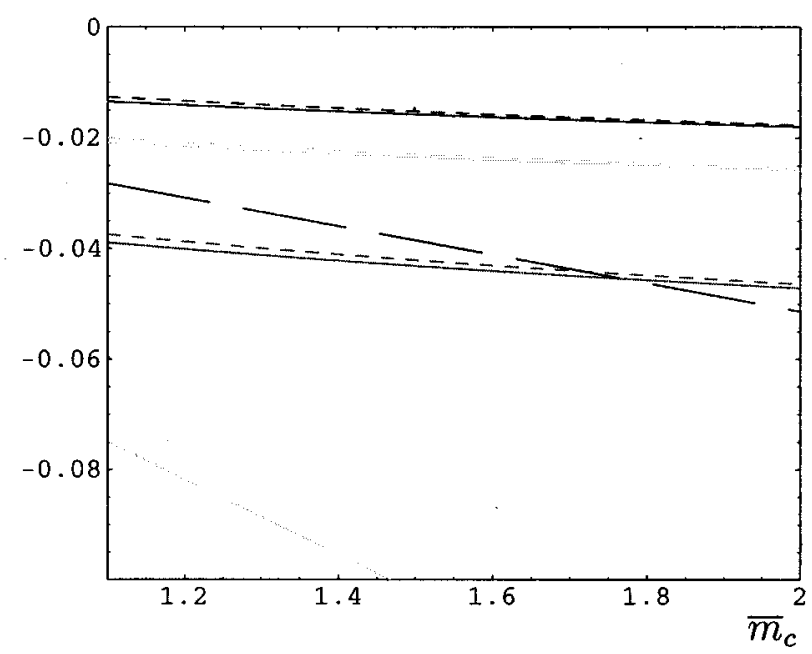

FIG. 3. $(\delta E)_{m_{c}}^{(1)}$ (continuous line), $(\delta E)_{m_{c} \rightarrow 0}^{(1)}$ (dashed line), $(\delta E)_{m_{c} \rightarrow \infty}^{(1)}$ (dotted line), as a function of $\bar{m}_{c}$ for $\bar{m}_{b}=4.201 \mathrm{GeV}$. Going down, the first set of lines corresponds to the $1 S$ state with $\mu=2.446 \mathrm{GeV}$, the second one to the $2 S$ state with $\mu$ $=1.065 \mathrm{GeV}$ and the third one to the $3 S$ state with $\mu$ $=0.724 \mathrm{GeV}$. Lines, which are not displayed, fall outside the plot range. The units are $\mathrm{GeV}$. 
TABLE I. $\left(\delta E_{b \bar{b}}\right)_{m_{c}}^{(1)},\left(\delta E_{b \bar{b}}\right)_{m_{c} \rightarrow 0}^{(1)}$ and $\left(\delta E_{b \bar{b}}\right)_{m_{c} \rightarrow \infty}^{(1)}$ for $\bar{m}_{b}=4.201 \mathrm{GeV}$ and $\bar{m}_{c}=1.237 \mathrm{GeV} ; \alpha_{\mathrm{s}}^{(4)}$ is calculated from $\Lambda \frac{(4)}{\mathrm{MS}}=0.292 \mathrm{GeV}$ at four loops. All dimensionful quantities are expressed in $\mathrm{GeV}$.

\begin{tabular}{ccccccc}
\hline \hline State & $\mu$ & $\alpha_{\mathrm{s}}^{(4)}(\mu)$ & $\bar{\rho}$ & $\left(\delta E_{b \bar{b}}\right)_{m_{c}}^{(1)}$ & $\left(\delta E_{b \bar{b}}\right)_{m_{c} \rightarrow 0}^{(1)}$ & $\left(\delta E_{b \bar{b}}\right)_{m_{c}}^{(1)} \rightarrow \infty$ \\
\hline $1^{3} S_{1}$ & 2.446 & 0.277 & 1.59 & -0.0143 & -0.032 & -0.0136 \\
$1^{3} P_{0}$ & 1.140 & 0.428 & 2.06 & -0.0210 & -0.076 & -0.0210 \\
$1^{3} P_{1}$ & 1.111 & 0.437 & 2.02 & -0.0221 & -0.079 & -0.0221 \\
$1^{3} P_{2}$ & 1.086 & 0.445 & 1.99 & -0.0232 & -0.082 & -0.0232 \\
$2^{3} S_{1}$ & 1.065 & 0.452 & 1.96 & -0.0219 & -0.084 & -0.0211 \\
$2^{3} P_{0}$ & 0.726 & 0.695 & 1.91 & -0.0426 & -0.199 & -0.0424 \\
$2^{3} P_{1}$ & 0.703 & 0.733 & 1.81 & -0.0490 & -0.222 & -0.0488 \\
$2^{3} P_{2}$ & 0.678 & 0.782 & 1.70 & -0.0581 & -0.252 & -0.0579 \\
$3^{3} S_{1}$ & 0.724 & 0.698 & 1.90 & -0.0405 & -0.201 & -0.0392 \\
\hline \hline
\end{tabular}

nium state (the dependence in Fig. 3 is only due to the different scales). In the region of interest $1 \mathrm{GeV} \lesssim \bar{m}_{c}$ $\lesssim 1.5 \mathrm{GeV},\left(\delta E_{b \bar{b}}\right)_{m_{c}}^{(1)}$ turns out to be very well approximated by $\left(\delta E_{b \bar{b}}\right)_{m_{c} \rightarrow \infty}^{(1)}$ (for all the levels), while $\left(\delta E_{b \bar{b}}\right)_{m_{c} \rightarrow 0}^{(1)}$ is far off. More specifically in Table I we list for all the levels at the above scales the values of $\left(\delta E_{b \bar{b}}\right)_{m_{c}}^{(1)},\left(\delta E_{b \bar{b}}\right)_{m_{c} \rightarrow 0}^{(1)}$ and $\left(\delta E_{b \bar{b}}\right)_{m_{c} \rightarrow \infty}^{(1)}$ with $\bar{m}_{c}=1.237 \mathrm{GeV}$ and $\bar{m}_{b}=4.201 \mathrm{GeV}$. The error of the "asymptotic approximation" $\left[\left(\delta E_{b \bar{b}}\right)_{m_{c} \rightarrow \infty}^{(1)}\right]$ is extremely small. In the worst situation (the $1 S$ level), it is about $5 \%$. In this case, however, the corrections are small (less than $1 \mathrm{MeV}$ ), so that they are beyond the accuracy of the present work. The "linear approximation" instead, at variance to what happens in the pole-mass expansion, is far off the exact values for all the states. This is in accordance with the general arguments of [7]. Let us notice, as a general remark, that the "asymptotic approximation" for the energy levels, works better than the "linear approximation" for the pole-mass expansion. We shall further comment on the above results in Sec. III C.

In Fig. 4 we show $(\delta E)_{m_{c}}^{(1)}$ (continuous line), and $(\delta E)_{m_{c} \rightarrow \infty}^{(1)}$ (dotted line), as a function of $\mu$ for $\bar{m}_{b}$ $=4.201 \mathrm{GeV}$ and $\bar{m}_{c}=1.237 \mathrm{GeV}$ for all the bottomonium states. Also this figure confirms that $(\delta E)_{m_{c} \rightarrow \infty}^{(1)}$ approximates $(\delta E)_{m_{c}}^{(1)}$ very well for all the levels.

\section{B. Order- $\varepsilon^{3}$ effects}

The $\left(\delta m_{b}\right)_{m_{c}}^{(2)}$ correction of Eq. (32) is not known exactly. However, following the calculation of the two-loop mass effects in the static potential in [22], in [7] $\left(\delta m_{b}\right)_{m_{c} \rightarrow 0}^{(2)}$ has been calculated:

$$
\begin{aligned}
\left(\delta m_{b}\right)_{m_{c} \rightarrow 0}^{(2)}= & \frac{\left(\alpha_{\mathrm{s}}^{(4)}\left(\bar{m}_{b}\right)\right)^{3}}{\pi} \bar{m}_{c}\left\{\frac{2}{9}+\frac{\beta_{0}^{(4)}}{12}\left(2 \ln \left(\frac{\bar{m}_{b}}{\bar{m}_{c}}\right)-4 \ln 2\right.\right. \\
& \left.\left.+\frac{14}{3}\right)-\frac{1}{9}\left(\frac{59}{15}+2 \ln 2\right)+\frac{19}{9 \pi}\left(f_{1} f_{2}+b_{1} b_{2}\right)\right\},
\end{aligned}
$$

where $f_{2}=0.470 \pm 0.005, \quad b_{2}=1.120 \pm 0.010,{ }^{8} \quad f_{1}=(\ln A$ $\left.-\ln b_{2}\right) /\left(\ln f_{2}-\ln b_{2}\right), b_{1}=\left(\ln A-\ln f_{2}\right) /\left(\ln b_{2}-\ln f_{2}\right)$ and $\ln A$ $=161 / 228+13 \zeta_{3} / 19-\ln 2$. For the values $\bar{m}_{b}=4.201 \mathrm{GeV}$ and $\bar{m}_{c}=1.237 \mathrm{GeV}$ we have

$$
\left(\delta m_{b}\right)_{m_{c} \rightarrow 0}^{(2)} \simeq 17 \mathrm{MeV} .
$$

From the analysis of the previous section we may expect that also this value approximates $\left(\delta m_{b}\right)_{m_{c}}^{(2)}$ with a relative uncertainty smaller than 20\%. In [7] it is argued that the sum $\left(\delta m_{b}\right)_{m_{c} \rightarrow 0}^{(1)}+\left(\delta m_{b}\right)_{m_{c} \rightarrow 0}^{(2)}$ may, indeed, approximate $\left(\delta m_{b}\right)_{m_{c}}^{(1)}+\left(\delta m_{b}\right)_{m_{c}}^{(2)}$ with an uncertainty of $10 \%$, while the precision of the sum $\left(\delta m_{b}\right)_{m_{c}}^{(1)}+\left(\delta m_{b}\right)_{m_{c} \rightarrow 0}^{(2)}$ is claimed to be worse. Finally, we notice that the series (32) shows no signals of convergence [compare with the figures of Eq. (36)].

The correction $\left(\delta E_{b \bar{b}}\right)_{m_{c}}^{(2)}$ of Eq. (31) is known only for the $1 S$ quarkonium state [7]. Using the analytic expression given in [7] at the values of the masses $\bar{m}_{b}=4.201 \mathrm{GeV}$, $\bar{m}_{c}=1.237 \mathrm{GeV}$ and at the scale $\mu=2.446 \mathrm{GeV}$, we obtain ${ }^{9}$

$$
\left(\delta E_{1 S}\right)_{m_{c}}^{(2)} \simeq-38.8 \mathrm{MeV}, \quad\left(\delta E_{1 S}\right)_{m_{c} \rightarrow \infty}^{(2)} \simeq-38.3 \mathrm{MeV}
$$

This confirms that the error of the "asymptotic approximation" is, indeed, extremely small [in the case of Eq. (42) it is about $1 \%$ ]. Considering that the $1 S$ state is the state located furthest from the "asymptotic" limit, we may conclude that the uncertainty connected with the use of the "asymptotic

\footnotetext{
${ }^{8}$ Somewhat larger errors are quoted in [23]. In our analysis only the central values are used.

${ }^{9} \mathrm{We}$ have used the $\overline{\mathrm{MS}}$ masses in the expression given in [7] in terms of the pole masses. This introduces at order $\varepsilon^{3}$ also a correction equal to $4 \alpha_{\mathrm{s}}^{(4)}(\mu) /(3 \pi)\left(\delta E_{b \bar{b}}\right)_{m_{c}}^{(1)}$ [see also Eq. (49) below]. This correction amounts to $\simeq-1.7 \mathrm{MeV}$ for the full $\left(\delta E_{b \bar{b}}\right)_{m}^{(1)}$ and to $\simeq-1.6 \mathrm{MeV}$ for the "asymptotic approximation" $\left(\delta E_{b \bar{b}}\right)_{m_{c} \rightarrow \infty}^{(1)}$.
} 

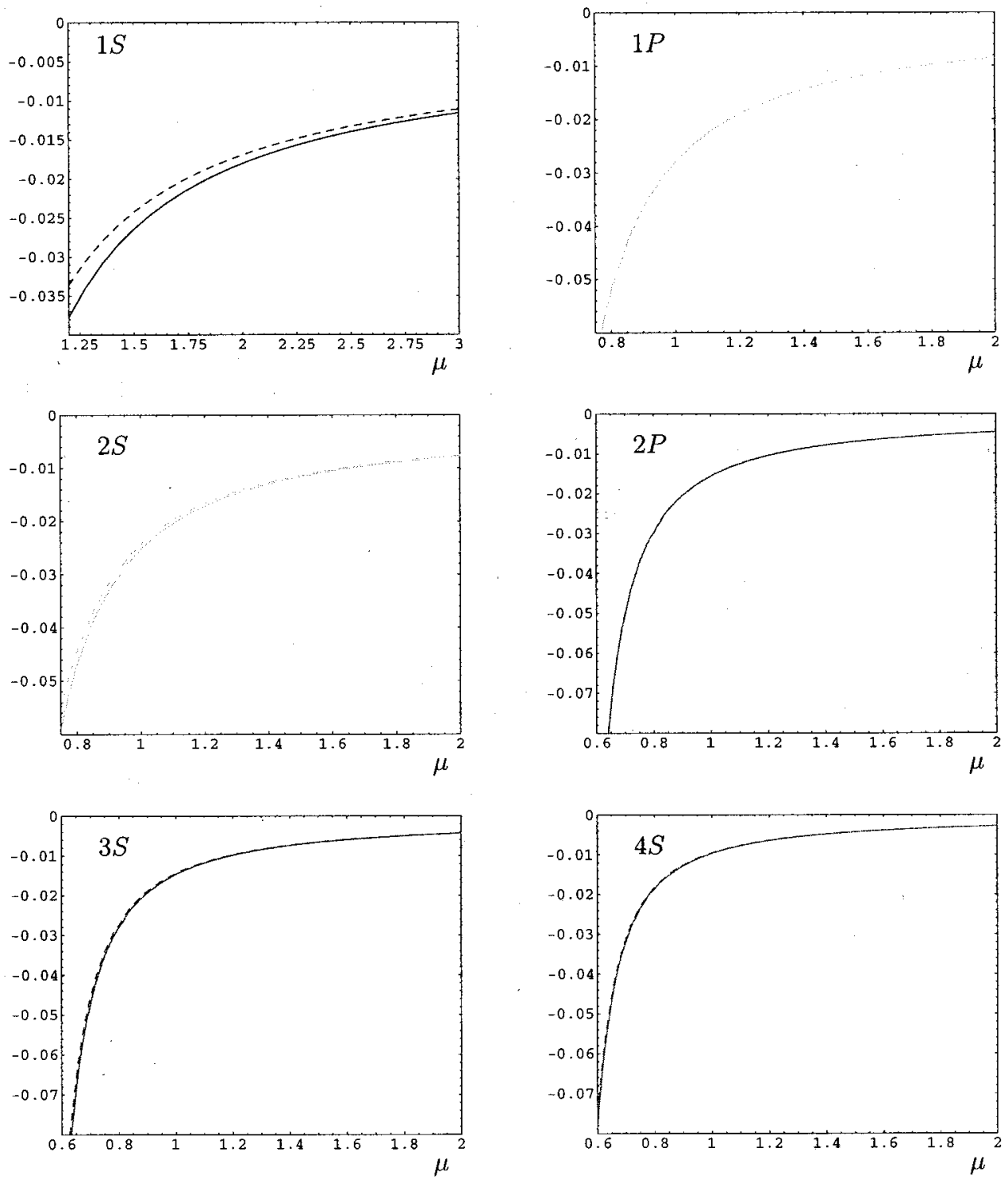

FIG. 4. $(\delta E)_{m_{c}}^{(1)}$ (continuous line), and $(\delta E)_{m_{c} \rightarrow \infty}^{(1)}$ (dotted line), as a function of $\mu$ for $\bar{m}_{b}=4.201 \mathrm{GeV}$ and $\bar{m}_{c}=1.237 \mathrm{GeV}$. The units are $\mathrm{GeV}$.

approximation" in the energy expansion is for all levels below the accuracy of the present work and, hence, negligible. The explicit expression for $\left(\delta E_{b} \bar{b}\right)_{m_{c} \rightarrow \infty}^{(2)}$ in terms of the pole mass is provided in Eq. (B3) of Appendix B.

\section{Discussion}

In this section we interpret and discuss the above results.

(a) The terms $\left(\delta m_{b}\right)_{m_{c}}^{(1)}$ and $\left(\delta m_{b}\right)_{m_{c}}^{(2)}$ are governed by the parameter $\xi$, which is the ratio between the charm and the bottom $\overline{\mathrm{MS}}$ masses. This is natural, since these are the only scales involved in the pole mass expansion. Since $\xi \sim 0.3$, which is smaller than 1 , the small $\xi$, or $m_{c}$, approximation is expected to work. On closer inspection of Eq. (33), we find

$$
\frac{\left(\delta m_{b}\right){ }_{m_{c}}^{(1)}-\left(\delta m_{b}\right)_{m_{c} \rightarrow 0}^{(1)}}{\left(\delta m_{b}\right)_{m_{c}}^{(1)}}=-\frac{6}{\pi^{2}} \xi+\mathcal{O}\left(\xi^{2}\right) \simeq-18 \%,
$$

which practically accounts for the entire difference between the first two terms in Eq. (36). Therefore, we may say that, concerning the pole mass expansion, the charm mass can be considered small and we are close to the situation of four active and massless flavors.

(b) The terms $\left(\delta E_{b \bar{b}}\right)_{m_{c}}^{(1)}$ and $\left(\delta E_{b \bar{b}}\right)_{m_{c}}^{(2)}$ are governed by the parameter $\bar{\rho}$. This is twice the ratio of the charm $\overline{\mathrm{MS}}$ mass and the typical momentum of $b$ and $\bar{b}$, which is the relevant scale of the bound state. This number turns out to be typically larger than 1 (see Table I). Therefore, an expansion 
in small $\bar{\rho}$, or $m_{c}$, is out of the question and only an expansion for large $\bar{\rho}$, or $m_{c}$ may work. From Eq. (37) we have

$$
\begin{aligned}
& \frac{\left(\delta E_{b \bar{b}}\right)_{m_{c}}^{(1)}-\left(\delta E_{b \bar{b}}\right)_{m_{c} \rightarrow \infty}^{(1)}}{\left(\delta E_{b \bar{b}}\right)_{m_{c}}^{(1)}} \\
& \sim-\frac{1}{\bar{\rho}^{2 l+2}} \frac{1}{2 \psi(n+l+1)-2 \psi(1)+5 / 3} .
\end{aligned}
$$

It follows that, even if the expansion parameter $\bar{\rho}$ is not particularly large, the "asymptotic approximation" turns out to work very well (for all the states), since the leading discarded term is suppressed as $1 / \bar{\rho}^{2 l+2}$, i.e. by at least two powers in $1 / \bar{\rho}$. The numerical factors contribute for extra suppressions. This explains why the data of Table I are reproduced so well by the "asymptotic approximation." Notice that, (i) states with higher $l$ are expected to be reproduced better by the asymptotic formula than states with lower $l$ (this can be checked explicitly in Table I by comparing the data for the $l=0$ states with those for the $l=1$ states, and in Fig. 4); (ii) states with higher $n$ are expected to be reproduced better than states with lower $n$ (as long as we are in the perturbative regime), since the parameter $\bar{\rho}$ grows like $n / \alpha_{\mathrm{s}}^{(4)}(\mu)$ [even if the growing of $\alpha_{\mathrm{s}}^{(4)}(\mu)$ partially compensates the growing of $n]$. In this sense the conclusions drawn in Sec. III B on the relative size of $\left(\delta E_{b \bar{b}}\right)_{m_{c}}^{(2)}$ with respect to $\left(\delta E_{b \bar{b}}\right)_{m_{c} \rightarrow \infty}^{(2)}$ for the $1 S$ state are expected to hold even better for the other (higher) states.

We may conclude that, where the energy-level expansion (in terms of the pole mass) is concerned, the charm mass can be considered large with respect to the typical scale of the bound state, so that the charm effectively decouples. Hence, charm-mass effects can be taken into account in the energylevel expansion of the bottomonium system (in terms of the pole mass) in a very effective way by considering it in the situation with three active and massless flavors only. ${ }^{10} \mathrm{We}$ have

$$
\begin{aligned}
\left(\delta E_{b \bar{b}}\right)_{m_{c 9}} \underset{\overline{5 \%}}{ }\left(\delta E_{b \bar{b}}\right)_{m_{c} \rightarrow \infty} & \\
= & E_{\mathrm{bin}, b \bar{b}}^{3}\left(\mu, \alpha_{\mathrm{s}}^{(3)}(\mu), m_{b, \text { pole }}\right) \\
& \quad-E_{\mathrm{bin}, b \bar{b}}^{4}\left(\mu, \alpha_{\mathrm{s}}^{(4)}(\mu), m_{b, \text { pole }}\right),
\end{aligned}
$$

where $E_{\text {bin }, b \bar{b}}^{n_{l}}$ are the bottomonium binding energies in terms of the bottom pole mass [Eq. (10)] with $n_{l}$ active and massless flavors only. Equation (45) is the key result of our analysis. It is expected to be valid at any order and it states that the charm decouples in the energy-level expansion (in terms of the pole mass) of the bottomonium system. Explicit expressions for Eq. (45) up to order $\varepsilon^{3}$ are given in Appendix B.

\section{Cross-check}

We have made a nontrivial cross-check of the major result, Eq. (45). We compared the total static energy of a $b \bar{b}$ system, $E_{\text {tot }}(r) \equiv 2 m_{b \text {, pole }}+V_{\mathrm{QCD}}(r)$, up to $\mathcal{O}\left(\alpha_{\mathrm{s}}^{3}\right)$, in the case where the full charm-mass corrections are included in $V_{\mathrm{QCD}}(r)$, the static potential in perturbative QCD, and in that where $V_{\mathrm{QCD}}(r)$ is evaluated in the limit $m_{c} \rightarrow \infty$. To be more precise, we re-analyzed the total energy $E_{\text {tot }}(r)$, studied in two hypothetical cases in [24]. We have included the full charm-mass corrections to the static QCD potential using the formulas obtained in $[22,7]$. Then we compared the total energy with the one calculated using $V_{\mathrm{OCD}}(r)$ in the limit $m_{c}$ $\rightarrow \infty$, i.e. with $n_{l}=3$. [In both cases, $\left(\delta m_{b}\right)_{m_{c}}^{(i)}$ is approximated by $\left.\left(\delta m_{b}\right)_{m_{c} \rightarrow 0}^{(i)}.\right]$ The total energies in the two cases agree with each other to a good accuracy: the difference is 3 $\mathrm{MeV}$ at $r=0.5 \mathrm{GeV}^{-1}$ and of order $0.1 \mathrm{MeV}$ in the range $1 \mathrm{GeV}^{-1}<r<3 \mathrm{GeV}^{-1}$. Since $E_{\text {tot }}(r)$ determines the bulk of the bottomonium spectrum [2], and since all the charmmass effects are included in it, this confirms the validity of our approximation. Note that the cross-check was made after cancellation of the leading renormalon contributions (expressing $m_{b \text {, pole }}$ in terms of $\bar{m}_{b}$ ), which adds a nontrivial point to the analyses done in the previous sections.

\section{E. Level expansions in terms of $\overline{\mathrm{MS}}$ masses}

A way to implement the above results in the bottomonium level expansion in terms of the $\overline{\mathrm{MS}}$ masses is to modify Eq. (23) into

$$
\begin{aligned}
E_{b \bar{b}}= & {\left[2 \bar{m}_{b}\left\{1+\frac{4}{3} \varepsilon \frac{\alpha_{\mathrm{s}}^{(4)}\left(\bar{m}_{b}\right)}{\pi}+\varepsilon^{2}\left(\frac{\alpha_{\mathrm{s}}^{(4)}\left(\bar{m}_{b}\right)}{\pi}\right)^{2} d_{1}^{(4)}+\varepsilon^{3}\left(\frac{\alpha_{\mathrm{s}}^{(4)}\left(\bar{m}_{b}\right)}{\pi}\right)^{3} d_{2}^{(4)}\right\}+E_{\mathrm{bin}, b \bar{b}}^{3} \mu, \alpha_{\mathrm{s}}^{(3)}(\mu), \bar{m}_{b}\left\{1+\frac{4}{3} \varepsilon \frac{\alpha_{\mathrm{s}}^{(4)}\left(\bar{m}_{b}\right)}{\pi}\right.\right.} \\
& \left.\left.\left.+\varepsilon^{2}\left(\frac{\alpha_{\mathrm{s}}^{(4)}\left(\bar{m}_{b}\right)}{\pi}\right)^{2} d_{1}^{(4)}\right\}\right)+\varepsilon^{2} 2\left(\delta m_{b}\right)_{m_{c}}^{(1)}+\varepsilon^{3}\left\{2\left(\delta m_{b}\right)_{m_{c}}^{(2)}-\frac{1}{4}\left(\frac{C_{F} \alpha_{\mathrm{s}}^{(4)}(\mu)}{n}\right)^{2}\left(\delta m_{b}\right)_{m_{c}}^{(1)}\right\}\right]_{\substack{\left.\alpha_{\mathrm{s}} \\
\alpha_{\mathrm{s}}^{(4)}=\bar{m}_{b}\right)=\mathrm{Eq} .(48) \\
(22)}}^{(46)} \\
\equiv & 2 \bar{m}_{b}+E_{b \bar{b}}^{(1)}\left(\mu, \alpha_{\mathrm{s}}^{(3)}(\mu), \bar{m}_{c}, \bar{m}_{b}\right) \varepsilon+E_{b \bar{b}}^{(2)}\left(\mu, \alpha_{\mathrm{s}}^{(3)}(\mu), \bar{m}_{c}, \bar{m}_{b}\right) \varepsilon^{2}+E_{b \bar{b}}^{(3)}\left(\mu, \alpha_{\mathrm{s}}^{(3)}(\mu), \bar{m}_{c}, \bar{m}_{b}\right) \varepsilon^{3}+\cdots,
\end{aligned}
$$

\footnotetext{
${ }^{10}$ It is worth while to stress that the situation here is the exact opposite with respect to that of the pole-mass expansion in terms of the $\overline{\mathrm{MS}}$ mass discussed in (a).
} 
where $\alpha_{\mathrm{s}}^{(4)}\left(\bar{m}_{b}\right)$ in Eq. (46) is understood as expanded everywhere around the scale $\mu$ according to Eq. (22) and around the coupling with 3 active massless flavors according to the following equation [21]:

$$
\alpha_{\mathrm{s}}^{(4)}(\mu)=\alpha_{\mathrm{s}}^{(3)}(\mu)\left\{1+\varepsilon \frac{\alpha_{\mathrm{s}}^{(3)}(\mu)}{3 \pi} \ln \left(\frac{\mu}{\bar{m}_{c}}\right)+\varepsilon^{2}\left(\frac{\alpha_{\mathrm{s}}^{(3)}(\mu)}{\pi}\right)^{2}\left[\frac{1}{9} \ln ^{2}\left(\frac{\mu}{\bar{m}_{c}}\right)+\frac{19}{12} \ln \left(\frac{\mu}{\bar{m}_{c}}\right)-\frac{11}{72}\right]\right\} .
$$

Both expansions are needed in order to have the same coupling in the pole mass and in the binding energy expansion so that the $\mathcal{O}\left(\Lambda_{\mathrm{QCD}}\right)$ renormalon cancellation is made explicit. Finite charm-mass corrections affect the pole-mass expansion and are explicitly written in the last line of Eq. (46).

Another possibility is to express also the binding energy at four massless flavors and to calculate the corrections to it. In this case Eq. (23) is modified into

$$
\begin{aligned}
& E_{b \bar{b}}=\left[2 \bar{m}_{b}\left\{1+\frac{4}{3} \varepsilon \frac{\alpha_{\mathrm{s}}^{(4)}\left(\bar{m}_{b}\right)}{\pi}+\varepsilon^{2}\left(\frac{\alpha_{\mathrm{s}}^{(4)}\left(\bar{m}_{b}\right)}{\pi}\right)^{2} d_{1}^{(4)}+\varepsilon^{3}\left(\frac{\alpha_{\mathrm{s}}^{(4)}\left(\bar{m}_{b}\right)}{\pi}\right)^{3} d_{2}^{(4)}\right\}+E_{\mathrm{bin}, b \bar{b}}^{4}\left(\mu, \alpha_{\mathrm{s}}^{(4)}(\mu), \bar{m}_{b}\left\{1+\frac{4}{3} \varepsilon \frac{\alpha_{\mathrm{s}}^{(4)}\left(\bar{m}_{b}\right)}{\pi}\right.\right.\right. \\
& \left.\left.+\varepsilon^{2}\left(\frac{\alpha_{\mathrm{s}}^{(4)}\left(\bar{m}_{b}\right)}{\pi}\right)^{2} d_{1}^{(4)}\right\}\right)+\varepsilon^{2}\left\{2\left(\delta m_{b}\right)_{m_{c}}^{(1)}+\left(\delta E_{b \bar{b}}\right)_{m_{c}}^{(1)}\left(\bar{m}_{b}, \bar{\rho}\right)\right\}+\varepsilon^{3}\left\{2\left(\delta m_{b}\right)_{m_{c}}^{(2)}-\frac{1}{4}\left(\frac{C_{F} \alpha_{\mathrm{s}}^{(4)}(\mu)}{n}\right)^{2}\left(\delta m_{b}\right)_{m_{c}}^{(1)}\right. \\
& \left.\left.+\left(\delta E_{b \bar{b}}\right)_{m_{c}}^{(2)}\left(\bar{m}_{b}, \bar{\rho}\right)+\frac{4}{3} \frac{\alpha_{\mathrm{s}}^{(4)}(\mu)}{\pi}\left(\delta E_{b \bar{b}}\right)_{m_{c}}^{(1)}\left(\bar{m}_{b}, \bar{\rho}\right)\right\}\right]_{\alpha_{\mathrm{s}}\left(\bar{m}_{b}\right)=\text { Eq. (22) }} \\
& \equiv 2 \bar{m}_{b}+E_{b \bar{b}}^{(1)}\left(\mu, \alpha_{\mathrm{s}}^{(4)}(\mu), \bar{m}_{c}, \bar{m}_{b}\right) \varepsilon+E_{b \bar{b}}^{(2)}\left(\mu, \alpha_{\mathrm{s}}^{(4)}(\mu), \bar{m}_{c}, \bar{m}_{b}\right) \varepsilon^{2}+E_{b \bar{b}}^{(3)}\left(\mu, \alpha_{\mathrm{s}}^{(4)}(\mu), \bar{m}_{c}, \bar{m}_{b}\right) \varepsilon^{3}+\cdots,
\end{aligned}
$$

where we have made use of the fact that $\rho=\bar{\rho}\left(1+\mathcal{O}\left(\alpha_{\mathrm{s}}^{2}\right)\right)$, and where $\alpha_{\mathrm{s}}^{(4)}\left(\bar{m}_{b}\right)$ in Eq. (49) is understood as expanded everywhere around the scale $\mu$ according to Eq. (22). The first two lines of Eq. (49) exactly correspond to Eq. (23), when calculated in the bottomonium case with four massless flavors.

Let us summarize our present knowledge of the different pieces of Eqs. (46) and (49).

(A) $E_{\mathrm{bin}, b \bar{b}}^{n_{l}}$ are the bottomonium binding energies in terms of the $\overline{\mathrm{MS}}$ masses with $n_{l}$ active and massless flavors only (i.e. $\left.m_{c}=0\right)$. They can be read from Eq. (10). A study of these contributions alone can be found in Ref. [2].

(B) $\left(\delta m_{b}\right)_{m_{c}}^{(1)}$ is given in Eq. (33). If we expand $\alpha_{\mathrm{s}}^{(4)}\left(\bar{m}_{b}\right)$ around the scale $\mu$, we then have to apply the replacement of Eq. (22). If we expand $\alpha_{\mathrm{s}}^{(4)}(\mu)$ around $\alpha_{\mathrm{s}}^{(3)}(\mu)$, we then have to apply also the replacement of Eq. (48). The quantity $\left(\delta m_{b}\right)_{m_{c}}^{(2)}$ is known only in the small $m_{c}$ limit and is given in Eq. (40). The use of $\left(\delta m_{b}\right)_{m_{c} \rightarrow 0}^{(2)}$ instead of $\left(\delta m_{b}\right)_{m_{c}}^{(2)}$ should account for $80 \%$ of the effect. In [7] it is claimed that the simultaneous use of $\left(\delta m_{b}\right)_{m_{c} \rightarrow 0}^{(1)}$ and $\left(\delta m_{b}\right)_{m_{c} \rightarrow 0}^{(2)}$ should account for $90 \%$ of the actual effect.

(C) $\left(\delta E_{b \bar{b}}\right)_{m_{c}}^{(1)}\left(\bar{m}_{b}, \bar{\rho}\right)$ is given in Eq. (37). The large $m_{c}$ limit of this expression is given in Eq. (39) and practically accounts for the full effect. The term $\left(\delta E_{b}\right)_{m_{c}}^{(2)}\left(\bar{m}_{b}, \bar{\rho}\right)$ has been calculated here in the large $m_{c}$ limit and is given in Eq. (B3). For the reasons discussed in Secs. III C and III D, we believe that Eq. (B3) accounts for practically the full effect.

Since Eq. (46) accounts explicitly for the decoupling of the charm quark at all orders in the binding-energy expansion (in terms of the pole mass), we expect results in this case to be more stable and reliable than by using Eq. (49). This will be confirmed by our numerical analysis. Therefore, we shall use Eq. (46) as our reference calculation (Sec. IV B). Equation (49) will be used in our analysis of theoretical uncertainties (Sec. IV C). Moreover, in our reference calculation of Sec. IV B we will approximate both $\left(\delta m_{b}\right)_{m_{c}}^{(1)}$ and $\left(\delta m_{b}\right)_{m_{c}}^{(2)}$ by means of the "linear approximations" $\left(\delta m_{b}\right)_{m_{c} \rightarrow 0}^{(1)}$ and $\left(\delta m_{b}\right)_{m_{c} \rightarrow 0}^{(2)}$. This proves to be slightly more stable than the use of the full correction $\left(\delta m_{b}\right)_{m_{c}}^{(1)}$. We take into account the full correction in the analysis of the uncertainties in Sec. IV C.

We conclude this section by giving the expression, which substitutes Eq. (23) in the $B_{c}$ case, when finite charm-mass effects are taken into account: 


$$
\begin{aligned}
E_{B_{c}}= & \bar{m}_{c}\left\{1+\frac{4}{3} \varepsilon \frac{\alpha_{\mathrm{s}}^{(3)}\left(\bar{m}_{c}\right)}{\pi}+\varepsilon^{2}\left(\frac{\alpha_{\mathrm{s}}^{(3)}\left(\bar{m}_{c}\right)}{\pi}\right)^{2} d_{1}^{(3)}+\varepsilon^{3}\left(\frac{\alpha_{\mathrm{s}}^{(3)}\left(\bar{m}_{c}\right)}{\pi}\right)^{3} d_{2}^{(3)}\right\}+\bar{m}_{b}\left\{1+\frac{4}{3} \varepsilon \frac{\alpha_{\mathrm{s}}^{(4)}\left(\bar{m}_{b}\right)}{\pi}+\varepsilon^{2}\left(\frac{\alpha_{\mathrm{s}}^{(4)}\left(\bar{m}_{b}\right)}{\pi}\right)^{2} d_{1}^{(4)}\right. \\
& \left.+\varepsilon^{3}\left(\frac{\alpha_{\mathrm{s}}^{(4)}\left(\bar{m}_{b}\right)}{\pi}\right)^{3} d_{2}^{(4)}\right\}+E_{\mathrm{bin}, B_{c}}^{3}\left(\mu, \alpha_{\mathrm{s}}^{(3)}(\mu), \bar{m}_{c}\left\{1+\frac{4}{3} \varepsilon \frac{\alpha_{\mathrm{s}}^{(3)}\left(\bar{m}_{c}\right)}{\pi}+\varepsilon^{2}\left(\frac{\alpha_{\mathrm{s}}^{(3)}\left(\bar{m}_{c}\right)}{\pi}\right)^{2} d_{1}^{(3)}\right\}, \bar{m}_{b}\left\{1+\frac{4}{3} \varepsilon \frac{\alpha_{\mathrm{s}}^{(4)}\left(\bar{m}_{b}\right)}{\pi}\right.\right. \\
& \left.\left.\left.+\varepsilon^{2}\left(\frac{\alpha_{\mathrm{s}}^{(4)}\left(\bar{m}_{b}\right)}{\pi}\right)^{2} d_{1}^{(4)}\right\}\right)+\varepsilon^{2}\left(\delta m_{b}\right)_{m_{c}}^{(1)}+\varepsilon^{3}\left\{\left(\delta m_{b}\right)_{m_{c}}^{(2)}-\frac{1}{2}\left(\frac{C_{F} \alpha_{\mathrm{s}}^{(4)}(\mu)}{n}\right)^{2}\left(\frac{\bar{m}_{c}}{\bar{m}_{c}+\bar{m}_{b}}\right)^{2}\left(\delta m_{b}\right)_{m_{c}}^{(1)}\right\}\right]_{\alpha_{\mathrm{s}}\left(\bar{m}_{c, b}\right)=\text { Eq. (22) }}^{\alpha_{\mathrm{s}}^{(4)}=\text { Eq. (48) }} \\
\equiv & \bar{m}_{c}+\bar{m}_{b}+E_{B_{c}}^{(1)}\left(\mu, \alpha_{\mathrm{s}}^{(3)}(\mu), \bar{m}_{c}, \bar{m}_{b}\right) \varepsilon+E_{B_{c}}^{(2)}\left(\mu, \alpha_{\mathrm{s}}^{(3)}(\mu), \bar{m}_{c}, \bar{m}_{b}\right) \varepsilon^{2}+E_{B_{c}}^{(3)}\left(\mu, \alpha_{\mathrm{s}}^{(3)}(\mu), \bar{m}_{c}, \bar{m}_{b}\right) \varepsilon^{3}+\cdots,
\end{aligned}
$$

where $\alpha_{\mathrm{s}}^{(3)}\left(\bar{m}_{c}\right)$ and $\alpha_{\mathrm{s}}^{(4)}\left(\bar{m}_{b}\right)$ in Eq. (51) are understood as expanded everywhere around the scale $\mu$ according to Eq. (22) and $\alpha_{\mathrm{s}}^{(4)}(\mu)$ around $\alpha_{\mathrm{s}}^{(3)}(\mu)$ according to Eq. (48).

\section{NUMERICAL ANALYSES}

In this section we examine numerically the series expansions of the bottomonium spectrum, Eqs. (46) and (49), and of the $B_{c}$ mass, Eq. (51).

\section{A. Scale-fixing procedures}

The quarkonium mass $E_{X}$ depends on the scale $\mu$, because of our incomplete knowledge of the perturbative series. We will fix the scale $\mu$ for each state $X$ in two different ways.

(A) We fix the scale $\mu=\mu_{X}^{A}$ by demanding stability of $E_{X}$ against variation of the scale:

$$
\left.\frac{d}{d \mu} E_{X}\left(\mu, \alpha_{\mathrm{s}}^{\left(n_{l}\right)}(\mu), \bar{m}_{i}\right)\right|_{\mu=\mu_{X}^{A}}=0 .
$$

(B) We fix the scale $\mu=\mu_{X}^{B}$ on the minimum of $\left|E_{X}^{(3)}\right|$ :

$$
\left.\frac{d}{d \mu}\left[E_{X}^{(3)}\left(\mu, \alpha_{\mathrm{s}}^{\left(n_{l}\right)}(\mu), \bar{m}_{i}\right)\right]^{2}\right|_{\mu=\mu_{X}^{B}}=0 .
$$

When we do this, we expect that the convergence properties of the series become optimal, and that the scale becomes close to the inverse of the physical size, $a_{X}$, of the bound state $X$ (defined as in Ref. [2]). If the scales fixed by Eq. (53) and Eq. (54) evidently do not fulfil these expectations, the theoretical predictions obtained in this way will be considered as unreliable. This typically happens when the coupling constant becomes larger than 1 . In the next section we will check if $\left|E_{X}^{(1)}\right|>\left|E_{X}^{(2)}\right|>\left|E_{X}^{(3)}\right|$ at the chosen scales. Notice that the criterion (B) of Eq. (54) practically always satisfies this request since $\min \left\{\left|E_{X}^{(3)}\right|\right\}=0$ in all the considered cases. This means that the results obtained using criterion (B) have to be taken with some caution. For this reason, in the following we will use them, rather than as an independent way to calculate the heavy-quarkonium spectra, as an independent check of the results obtained using criterion (A) as well as a means to determine the uncertainties related to the fixing of the scale $\mu_{X}$. Comparisons of the chosen scales and the bound-state sizes have been given in Sec. 5 of [2] and we do not repeat them here.

\section{B. Numerical results}

Here, we present results from both scale-fixing procedures on Eq. (46) for the bottomonium spectrum and on Eq. (51) for the $B_{c}$ mass. We calculate both $\left(\delta m_{b}\right)_{m_{c}}^{(1)}$ and $\left(\delta m_{b}\right)_{m_{c}}^{(2)}$ by means of the "linear approximations" $\left(\delta m_{b}\right)_{m_{c} \rightarrow 0}^{(1)}$ and $\left(\delta m_{b}\right)_{m_{c} \rightarrow 0}^{(2)}$. The coupling constant $\alpha_{\mathrm{s}}$ is defined as in Sec. II B. The uncertainties on $\alpha_{\mathrm{s}}^{(5)}\left(M_{Z}\right)$ [or on $\Lambda_{\overline{\mathrm{MS}}}$, see Eqs. (26) -(28)] are the only ones that we take into account here. Other sources of uncertainties and in particular those related to the finite charm-mass effects will be discussed in Sec. IV C.

\section{Scale-fixing procedure (A)}

Since we expect the ground states of the $b \bar{b}$ and $c \bar{c}$ systems to be the states least affected by nonperturbative corrections, we fix $\bar{m}_{b}$ and $\bar{m}_{c}$ through the two conditions

$$
\begin{gathered}
E_{\Upsilon(1 S)}\left(\mu_{X}^{A}, \alpha_{\mathrm{s}}^{(3)}\left(\mu_{X}^{A}\right), \bar{m}_{c}, \bar{m}_{b}\right)=E_{\Upsilon(1 S)}^{\exp }=9.460 \mathrm{GeV}, \\
E_{J / \psi}\left(\mu_{X}^{A}, \alpha_{\mathrm{s}}^{(3)}\left(\mu_{X}^{A}\right), \bar{m}_{c}\right)=E_{J / \psi}^{\exp }=3.097 \mathrm{GeV},
\end{gathered}
$$

where the experimental values of the vector ground states have been taken from [20]. We assume, for the moment, that this identification is not affected by nonperturbative corrections. From Eqs. (55), (56) and (53) we determine $\mu_{X}^{A}$ (see Table II), and the $b$ and $c \overline{\mathrm{MS}}$ masses:

$$
\begin{aligned}
& \bar{m}_{b}=4190_{+19}^{-20} \mathrm{MeV}, \\
& \bar{m}_{c}=1237_{+16}^{-16} \mathrm{MeV} .
\end{aligned}
$$


TABLE II. Theoretical predictions for the bottomonium and $B_{c}$ masses from Eqs. (46) and (51), respectively $\left[\left(\delta m_{b}\right)_{m_{c}}^{(1,2)} \simeq\left(\delta m_{b}\right)_{m_{c} \rightarrow 0}^{(1,2)}\right]$, when the scales $\mu_{X}^{A}$ are fixed through Eq. (53). The quantities $E_{X}^{(j)}$ are the order $\varepsilon^{j}$ contributions to the spectrum and are defined by Eqs. (47) and (52). The $c$ - and $b$-quark $\overline{\mathrm{MS}}$ masses are fixed on the experimental values of the $J / \psi$ and $\Upsilon(1 S)$ masses, respectively. The coupling constant $\alpha_{\mathrm{s}}^{(3)}$ has been defined according to Sec. II B. The uncertainties in the third and fourth columns refer to the uncertainties in $\alpha_{\mathrm{s}}^{(5)}\left(M_{Z}\right)$ only. All the other data refer to $\alpha_{\mathrm{s}}^{(5)}\left(M_{Z}\right)=0.1181$ and to the $\overline{\mathrm{MS}}$ quark masses fixed on the central values of Eqs. (58) and (57). All dimensionful numbers are in GeV.

\begin{tabular}{ccccccccc}
\hline \hline State $X$ & $E_{X}^{\exp }$ & $E_{X}$ & $E_{X}^{\exp }-E_{X}$ & $E_{X}^{(1)}$ & $E_{X}^{(2)}$ & $E_{X}^{(3)}$ & $\mu_{X}^{A}$ & $\alpha_{\mathrm{s}}^{(3)}\left(\mu_{X}^{A}\right)$ \\
\hline$\Upsilon\left(1^{3} S_{1}\right)$ & 9.460 & 9.460 & 0 & 0.866 & 0.208 & 0.006 & 2.14 & 0.286 \\
$\Upsilon\left(1^{3} P_{0}\right)$ & 9.860 & $9.995_{-62}^{+75}$ & $-0.135_{+62}^{-75}$ & 1.534 & 0.101 & -0.021 & 1.08 & 0.459 \\
$\Upsilon\left(1^{3} P_{1}\right)$ & 9.893 & $10.004_{-63}^{+78}$ & $-0.111_{+63}^{-78}$ & 1.564 & 0.081 & -0.022 & 1.05 & 0.468 \\
$\Upsilon\left(1^{3} P_{2}\right)$ & 9.913 & $10.012_{-65}^{+81}$ & $-0.099_{+65}^{-81}$ & 1.591 & 0.063 & -0.022 & 1.034 & 0.477 \\
$\Upsilon\left(2^{3} S_{1}\right)$ & 10.023 & $10.084_{-75}^{+93}$ & $-0.061_{+73}^{-93}$ & 1.618 & 0.096 & -0.010 & 1.02 & 0.486 \\
$\Upsilon\left(2^{3} P_{0}\right)$ & 10.232 & $10.548_{-151}^{+196}$ & $-0.316_{+151}^{-196}$ & 2.421 & -0.356 & 0.102 & 0.778 & 0.710 \\
$\Upsilon\left(2^{3} P_{1}\right)$ & 10.255 & $10.564_{-153}^{+200}$ & $-0.309_{+153}^{-200}$ & 2.472 & -0.404 & 0.116 & 0.770 & 0.726 \\
$\Upsilon\left(2^{3} P_{2}\right)$ & 10.268 & $10.578_{-155}^{+203}$ & $-0.310_{+155}^{-203}$ & 2.518 & -0.449 & 0.129 & 0.762 & 0.740 \\
$Y\left(3^{3} S_{1}\right)$ & 10.355 & $10.645_{-168}^{+218}$ & $-0.290_{+168}^{-218}$ & 2.472 & -0.348 & 0.140 & 0.770 & 0.726 \\
$\Upsilon\left(4^{3} S_{1}\right)$ & 10.580 & $*$ & $*$ & $*$ & $*$ & $*$ & $*$ & $*$ \\
$B_{c}\left(1^{1} S_{0}\right)$ & $6.4 \pm 0.4$ & $6.307_{-2}^{+4}$ & $0.1 \pm 0.4$ & 0.675 & 0.188 & 0.017 & 1.62 & 0.334 \\
\hline \hline
\end{tabular}

These values are in good agreement with the estimates based on $Y[25,15,26,7]$ and charmonium [27] sum rules respectively. The charm mass given in Eq. (58) corresponds to the one quoted in Table 2, column (i) of Ref. [2] [reported also in Eq. (30) of the present paper], since it is not affected in our analysis by massive quark-loop effects. The bottom mass given in Eq. (57) is new. The errors refer to the uncertainties in $\alpha_{\mathrm{s}}^{(5)}\left(M_{Z}\right)$ only.

Using these masses as input, we calculate the energy levels of other observed bottomonium and $B_{c}$ states. In Figs. 5-7 we display the $\mu$ dependence of the different energy levels $E_{X}$ measured from $2 \bar{m}_{b}$ and $\bar{m}_{b}+\bar{m}_{c}$, respectively (at different orders in $\varepsilon$ ) before scale fixing. The levels, after scale fixing by Eq. (53), are given in Table II. Theoretical predictions that we consider unreliable, in the sense specified in Sec. IV A, are not displayed and indicated with a star (*). Generally, for states that we consider reliably calculable in the perturbative approach, the scale dependence decreases as we include more terms of the perturbative series. For states whose predictions we consider unreliable, the series would become much more convergent if we chose a scale different from (typically larger than) $\mu_{X}$. The theoretical prediction for $B_{c}$ is consistent with the experimental value, although the experimental error is large. It is also in agreement with the determination of [16].

\section{Scale-fixing procedure (B)}

By fixing the bottom and charm $\overline{\mathrm{MS}}$ masses on the experimental values of the $Y(1 S)$ and $J / \psi$ masses via the scalefixing procedure of Eq. (54), respectively, we obtain

$$
\begin{gathered}
\bar{m}_{b}=4190_{+19}^{-20} \mathrm{MeV}, \\
\bar{m}_{c}=1241_{+14}^{-16} \mathrm{MeV} .
\end{gathered}
$$

These values are in agreement with the estimates based on the procedure (A), given in Eqs. (57) and (58). The errors refer to the uncertainties in $\alpha_{\mathrm{s}}^{(5)}\left(M_{Z}\right)$ only. Using these masses as input and fixing the scales through Eq. (54), we calculate the energy levels of other observed bottomonium and $B_{c}$ states. The levels are given in Table III. Reliable theoretical predictions agree well with those given in Table II.

Let us compare the present results for the bottomonium states with the corresponding results obtained in the $m_{c}=0$ case in Table 2 column (i) of [2]. The value of the bottom $\overline{\mathrm{MS}}$ mass $\bar{m}_{b}$ determined from the $\Upsilon(1 S)$ mass is lowered by about $11 \mathrm{MeV}$ by the inclusion of the nonzero charm-mass effects, for the same input value of $\alpha_{\mathrm{s}}^{(5)}\left(M_{Z}\right)$. The masses of the $n=2$ bottomonium states are raised by about $70-100$ $\mathrm{MeV}$ by the charm-mass effects; the masses of the $n=3$ states are raised by about $240-280 \mathrm{MeV}$ by these effects. These tendencies can be understood as originating from an increase of the binding energies in these systems: if $\mu_{X}^{A, B}$ $\lesssim m_{c}, \alpha_{\mathrm{s}}^{(3)}$ at the corresponding scale becomes larger than $\alpha_{\mathrm{s}}^{(4)}$; hence, the higher the energy level is, the more its mass is increased. For this reason, the value of the input $\alpha_{\mathrm{s}}^{(5)}\left(M_{Z}\right)$, which reproduces the whole level structure of the experimental data optimally, becomes smaller when we include the charm-mass effects: in the $m_{c}=0$ case, $\alpha_{\mathrm{s}}^{(5)}\left(M_{Z}\right)=0.1181$ reproduces the experimental data fairly well, if we take an average of the $S$-wave and $P$-wave levels corresponding to each principal quantum number $n$, while the agreement is better for $\alpha_{\mathrm{s}}^{(5)}\left(M_{Z}\right)=0.1161$ when the charm-mass effects are included. It also remains that the predictions for the $S-P$ splittings and the fine splittings are smaller than the experimental values, as noted in [2]. However, it should also be noted that for these observables the perturbative expansions considered here include only tree-level contributions. As for 

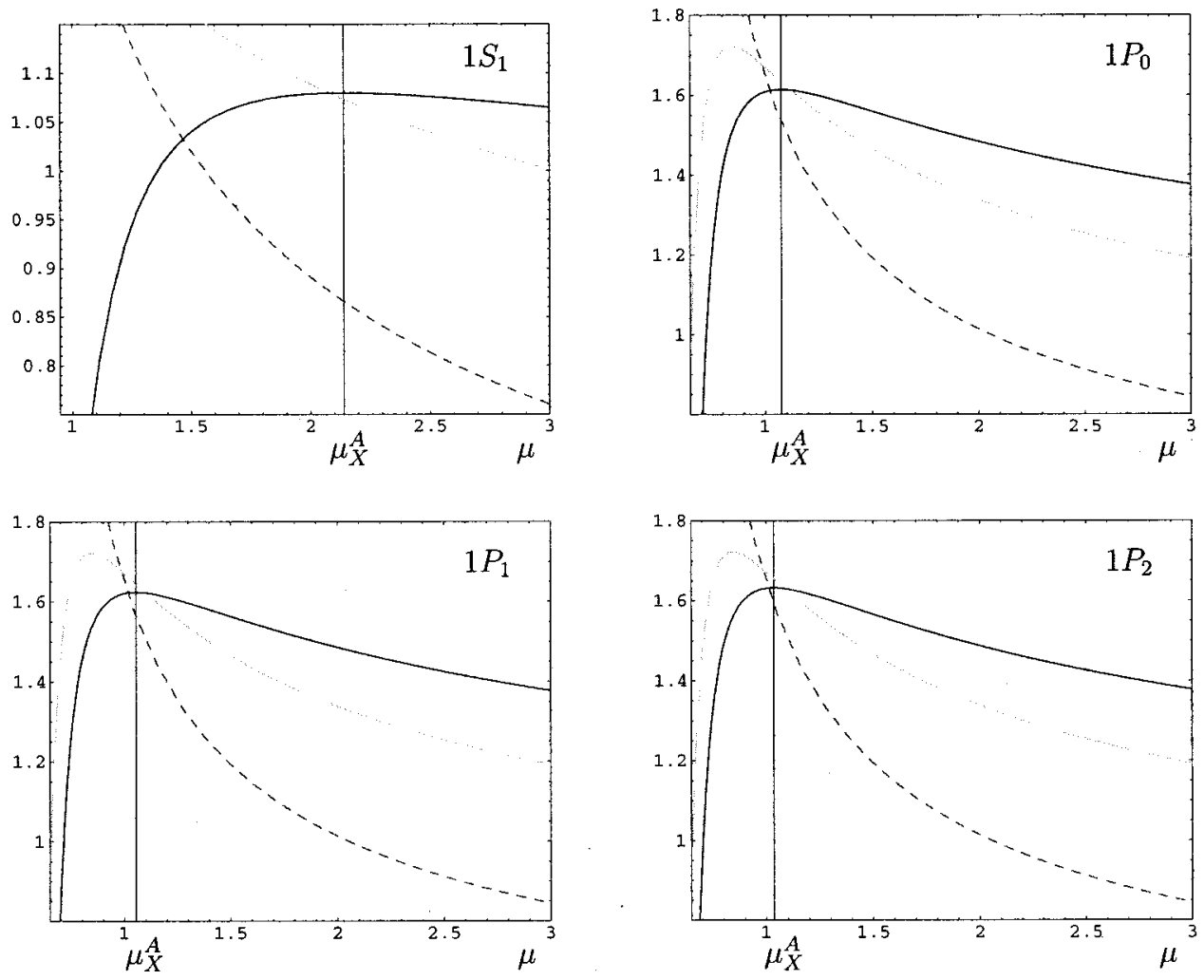

FIG. 5. $E_{b \bar{b}}^{(1)}$ (dotted line), $E_{b \bar{b}}^{(1)}+E_{b \bar{b}}^{(2)}$ (dashed line) and $E_{b \bar{b}}^{(1)}+E_{b \bar{b}}^{(2)}+E_{b \bar{b}}^{(3)}$ (continuous line), as defined in Eq. (47) $\left[\left(\delta m_{b}\right)_{m}^{(1,2)}\right.$ $\left.\simeq\left(\delta m_{b}\right)_{m_{c} \rightarrow 0}^{(1,2)}\right]$, versus $\mu$ for the states $1 S_{1}, 1 P_{0}, 1 P_{1}$ and $1 P_{2}$. The parameters are $\bar{m}_{b}=4.190 \mathrm{GeV}, \bar{m}_{c}=1.237 \mathrm{GeV}$ and $\Lambda \frac{(3)}{\mathrm{MS}}$ $=0.333 \mathrm{GeV} ; \mu_{X}^{A}$ obtained from the minimal sensitivity prescription, Eq. (53), is explicitly shown. The units are GeV.

the $B_{c}(1 S)$ mass, it is reduced by about $10-20 \mathrm{MeV}$ by the charm-mass effects, when $\bar{m}_{b}, \bar{m}_{c}$ and $\alpha_{\mathrm{s}}\left(M_{Z}\right)$ are taken as input.

\section{Error estimates}

In this section we take the results listed in Table II as our reference calculation and discuss different kinds of uncertainties that may affect them. Besides nonperturbative corrections, there are four kinds of uncertainties, which we list below.

(1) Uncertainties related to the charm-mass effects discussed in this paper. Since the charm-mass effects are the main subject of the present paper, their errors will be discussed in some detail. In evaluating the effects of charmmass loops in the perturbative expansion of the quarkonium levels, we have considered different options (see Sec. III). Here we estimate the differences between these options and the reference calculation in the previous section. This also shows that the reference calculation is the most reliable in the sense discussed at the beginning of Sec. IV. In Table IV the following error estimates are listed [the scales $\mu$ are fixed by Eq. (53); the quoted values correspond to $\alpha_{\mathrm{s}}^{(5)}\left(M_{Z}\right)$ $=0.1181 \pm 0.0020]$ : (i) The difference between the results obtained from Eq. (46) [Eq. (51) for $B_{c}$ ] when the full expression of $\left(\delta m_{b}\right)_{m_{c}}^{(1)}$, as given in Eq. (33), is used instead of the linear approximation $\left(\delta m_{b}\right)_{m_{c} \rightarrow 0}^{(1)}$ and the results of Table
II. (ii) The difference between the results obtained from Eq. (49) when $\left(\delta m_{b}\right)_{m_{c}}^{(1)}$ is approximated by $\left(\delta m_{b}\right)_{m_{c} \rightarrow 0}^{(1)}$ and the results for the bottomonium system of Table II. (iii) The difference between the results obtained from Eq. (49) when the full expression of $\left(\delta m_{b}\right)_{m_{c}}^{(1)}$ is used and the results for the bottomonium system of Table II.

(2) Uncertainty of $\alpha_{\mathrm{s}}^{(5)}\left(M_{Z}\right)$. This has been taken into account in the results presented in the previous section and listed in Tables II and III.

(3) Uncertainties from higher-order corrections. These have already been considered in [2] and may be estimated as follows (see Table V): (i) The difference between the theoretical predictions computed using $\alpha_{\mathrm{s}}(\mu)$ as obtained by solving the renormalization-group equation numerically at 4 loops and the definition of $\alpha_{\mathrm{s}}(\mu)$ used in the previous sections and specified in Sec. II B. (ii) The contributions $\pm\left|E_{X}^{(3)}\right|$ from Table II $\left( \pm\left|E_{X}^{(3)}\right| / 2\right.$ for $\left.\delta \bar{m}_{b}\right)$. We have not explicitly considered here uncertainties related to different implementations of the $\varepsilon$-expansion scheme. Indeed, one could imagine different ways of counting in $\varepsilon$ terms that are not directly related to the renormalon cancellation in the pole mass and in the static potential, such as relativistic corrections or spin-orbit and spin-spin interaction terms. A naive estimate, obtained by subtracting from the definition of $E_{X}^{(3)}$ given in Eq. (47) all the terms mentioned above, gives in the $\bar{m}_{b}$ case $\pm \mid E_{X}^{(3)}$ (after reshuffling) $\mid / 2= \pm 25 \mathrm{MeV}$. However, 

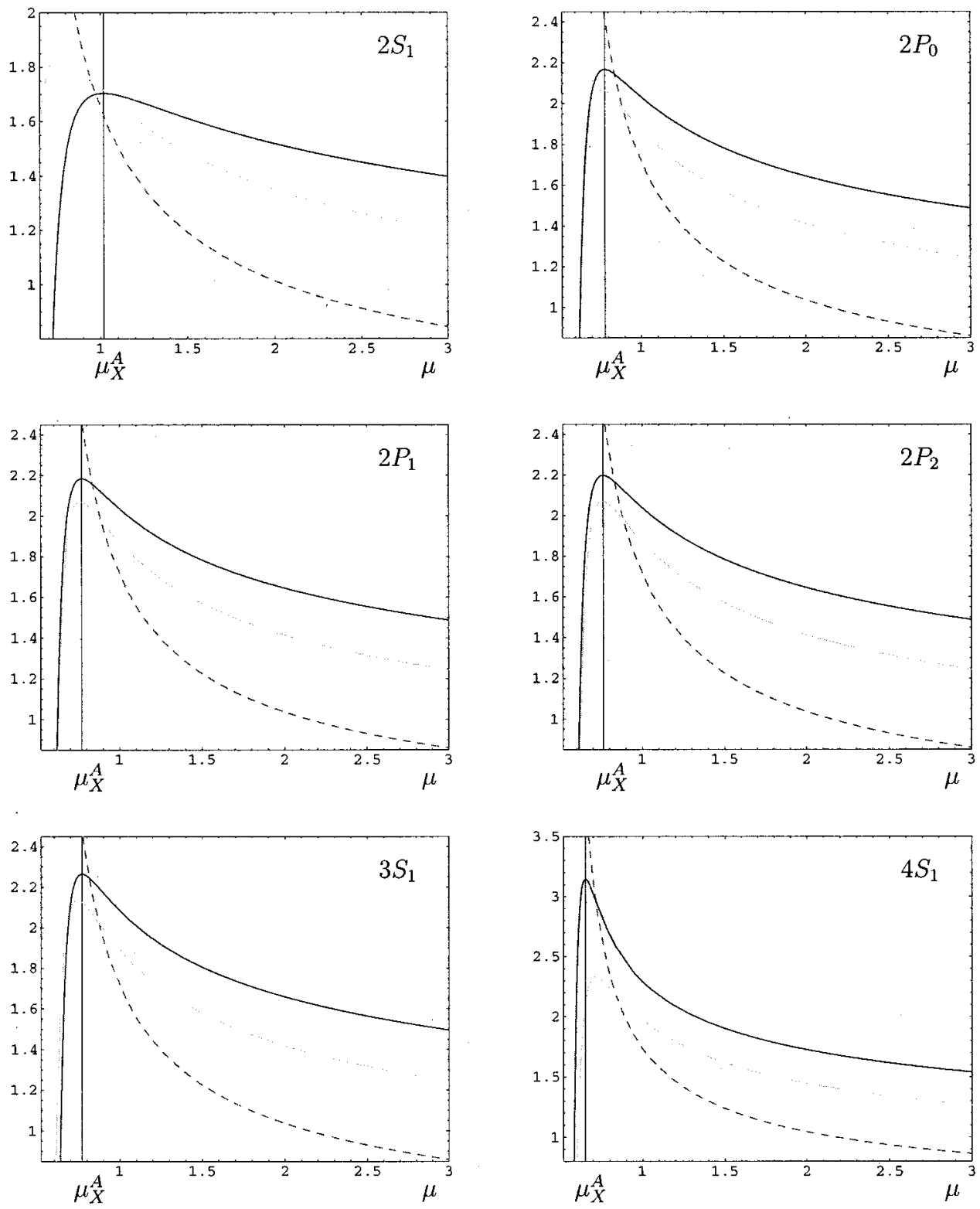

FIG. 6. $E_{b \bar{b}}^{(1)}$ (dotted line), $E_{b \bar{b}}^{(1)}+E_{b \bar{b}}^{(2)}$ (dashed line) and $E_{b \bar{b}}^{(1)}+E_{b \bar{b}}^{(2)}+E_{b \bar{b}}^{(3)}$ (continuous line), as defined in Eq. (47) versus $\mu$ for the states $2 S_{1}, 2 P_{0}, 2 P_{1}, 2 P_{2}, 3 S_{1}$ and $4 S_{1}$. Other notations are the same as in Fig. 5.

further investigations are needed on this point. In [2] also the difference between the theoretical predictions computed using the 3-loop and the 4-loop running coupling constants has been considered. The size of these uncertainties is typically smaller than those listed here, and, therefore, they are not considered.

(4) Uncertainties in the scale-fixing procedure. These are also part of the higher-order corrections and may be estimated as follows (see Table VI): (i) The difference between the theoretical estimates of Table III obtained by fixing $\mu_{X}$ $=\mu_{X}^{B}$ via the condition (54) and the results of Table II, obtained by fixing $\mu_{X}=\mu_{X}^{A}$ via the condition (53). (ii) For comparison with the error estimates given in conventional analyses, we consider the (maximal) variations of $\bar{m}_{b}$ and $E_{X}$ when we fix the scale as $\pm 10 \%$ of the minimal sensitivity scale: $\mu=\mu_{X}^{A} \times(1 \pm 0.1)$, where $\mu_{X}^{A}$ is taken from Table II.
This is numerically close to the scale-fixing uncertainty evaluation done in [12].

A summary of the above uncertainties is given in Table VII. The figures are obtained as \pm the absolute value of the maximum of the corresponding uncertainties. In the column labeled "h.o. corrections" the maximum is taken from the uncertainties of Tables V and VI. Note that in Table V we have not calculated the error associated with different implementations of the $\varepsilon$-expansion for levels higher than the ground state.

\section{Charm-mass corrections}

In Table VIII we display the pure charm-mass corrections of order $\varepsilon^{2}$,

$$
2\left(\delta m_{b}\right)_{m_{c} \rightarrow 0}^{(1)}+\left(\delta E_{b \bar{b}}\right)_{m_{c} \rightarrow \infty}^{(1)}\left(\bar{m}_{b}, \bar{\rho}\right)
$$




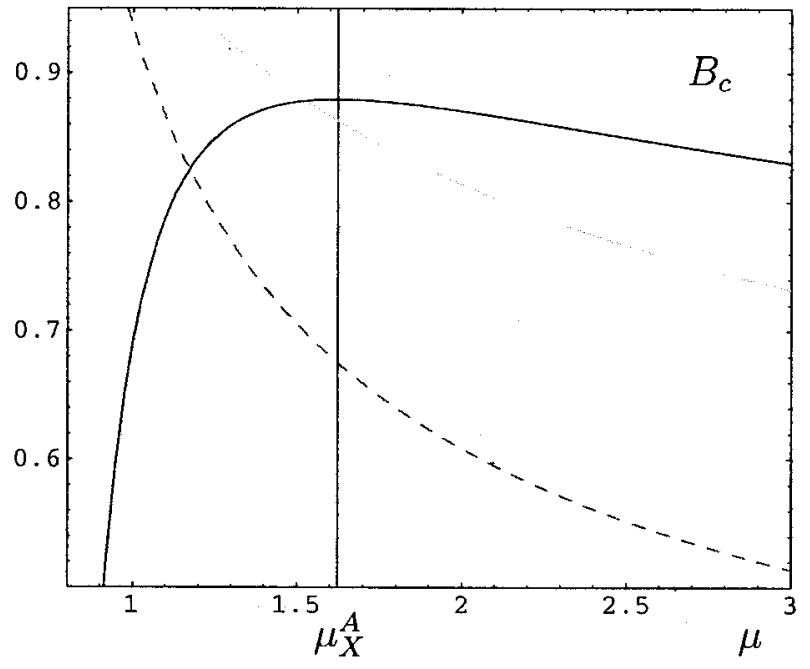

FIG. 7. $E_{B_{c}}^{(1)}$ (dotted line), $E_{B_{c}}^{(1)}+E_{B_{c}}^{(2)}$ (dashed line) and $E_{B_{c}}^{(1)}$ $+E_{B_{c}}^{(2)}+E_{B_{c}}^{(3)}$ (continuous line), as defined in Eq. (52) versus $\mu$ for the $B_{c}$. Other notations are the same as in Fig. 5.

and of order $\varepsilon^{3}$,

$$
\begin{gathered}
2\left(\delta m_{b}\right)_{m_{c} \rightarrow 0}^{(2)}-\frac{1}{4}\left(\frac{C_{F} \alpha_{\mathrm{s}}^{(4)}(\mu)}{n}\right)^{2}\left(\delta m_{b}\right)_{m_{c} \rightarrow 0}^{(1)} \\
+\left(\delta E_{b \bar{b}}\right)_{m_{c} \rightarrow \infty}^{(2)}\left(\bar{m}_{b}, \bar{\rho}\right) \\
+\frac{4}{3} \frac{\alpha_{\mathrm{s}}^{(4)}(\mu)}{\pi}\left(\delta E_{b \bar{b}}\right)_{m_{c} \rightarrow \infty}^{(1)}\left(\bar{m}_{b}, \bar{\rho}\right),
\end{gathered}
$$

of Eq. (49), where the energy is expressed in terms of the 4-flavor coupling. The full energy levels can be easily obtained from the data displayed in column (ii) of Table IV and from Table II. Table VIII shows that also the disentangled
TABLE IV. Differences between the determination (i), (ii) and (iii) discussed in the item (1) of Sec. IV C and the theoretical predictions of Table II. The central values correspond to $\alpha_{\mathrm{s}}^{(5)}\left(M_{Z}\right)$ $=0.1181$, the upper values to $\alpha_{\mathrm{s}}^{(5)}\left(M_{Z}\right)=0.1201$, the lower values to $\alpha_{\mathrm{s}}^{(5)}\left(M_{Z}\right)=0.1161$. The scale is fixed according to the procedure (A) of Eq. (53). Those values corresponding to unreliable theoreti-

\begin{tabular}{|c|c|c|c|c|}
\hline & \multicolumn{3}{|c|}{ Uncertainties of charm-mass effects } \\
\hline & & (i) & (ii) & (iii) \\
\hline & $\delta \bar{m}_{b}$ & $+1_{-0}^{+0}$ & $-3_{-0}^{+1}$ & $-2_{-0}^{+1}$ \\
\hline \multirow{10}{*}{$\delta E_{X}$} & $Y\left(1^{3} P_{0}\right)$ & $+10_{-1}^{+2}$ & $-15_{+3}^{-6}$ & $-5_{+2}^{-5}$ \\
\hline & $Y\left(1^{3} P_{1}\right)$ & $+12_{-2}^{+2}$ & $-15_{+2}^{-7}$ & $-4_{+1}^{-5}$ \\
\hline & $Y\left(1^{3} P_{2}\right)$ & $+14_{-2}^{+1}$ & $-15_{+2}^{-9}$ & $-3_{+1}^{-6}$ \\
\hline & $Y\left(2^{3} S_{1}\right)$ & $+14_{-2}^{+2}$ & $-18_{+3}^{-9}$ & $-4_{+1}^{-7}$ \\
\hline & $Y\left(2^{3} P_{0}\right)$ & $+77_{+2}^{+5}$ & $-12_{*}^{-29}$ & $*$ \\
\hline & $Y\left(2^{3} P_{1}\right)$ & $+85_{+6}^{+5}$ & $-4_{*}^{-33}$ & $*$ \\
\hline & $Y\left(2^{3} P_{2}\right)$ & $+93_{+14}^{+4}$ & $+6_{*}^{*} 39$ & $*$ \\
\hline & $Y\left(3^{3} S_{1}\right)$ & $+79_{-1}^{+7}$ & $-19_{+24}^{-30}$ & $+87_{*}^{-35}$ \\
\hline & $Y\left(4^{3} S_{1}\right)$ & $*$ & $*$ & $*$ \\
\hline & $B_{c}\left(1^{1} S_{0}\right)$ & $+1_{-1}^{-1}$ & - & - \\
\hline
\end{tabular}
cal predictions are indicated with a star $(*)$. All dimensionful numbers are in $\mathrm{MeV}$.

finite charm-mass corrections exhibit a reasonable convergence, in correspondence to the reliable predictions of Table II.

\section{CONCLUSIONS AND DISCUSSION}

We have computed the bottomonium spectrum within the framework of perturbative QCD, including the nonzero charm-mass effects. The effects of a charm mass $m_{c}>0$ as compared to the $m_{c}=0$ case are to increase the level spacings; the effects are larger among the higher levels. They can be understood as follows: the effective coupling becomes larger at the relevant scale when the decoupling of the charm quark is incorporated; consequently, the binding energy increases. Since we fixed $\bar{m}_{b} \equiv m_{b}^{\overline{\mathrm{MS}}}\left(m_{b}^{\overline{\mathrm{MS}}}\right)$ on $\Upsilon(1 S)$ in our analysis, the net effects are to decrease $\bar{m}_{b}$ by about $11 \mathrm{MeV}$

TABLE III. Theoretical predictions for the charmonium, bottomonium and $B_{c}$ masses, when the scales $\mu_{X}^{B}$ are fixed through Eq. (54). The other conventions are the same as in Table II.

\begin{tabular}{cccccccc}
\hline \hline State $X$ & $E_{X}^{\exp }$ & $E_{X}$ & $E_{X}^{(1)}$ & $E_{X}^{(2)}$ & $E_{X}^{(3)}$ & $\mu_{X}^{B}$ & $\alpha_{\mathrm{s}}^{(3)}\left(\mu_{X}^{B}\right)$ \\
\hline$\Upsilon\left(1^{3} S_{1}\right)$ & 9.460 & 9.460 & 0.876 & 0.204 & 0 & 2.08 & 0.290 \\
$\Upsilon\left(1^{3} P_{0}\right)$ & 9.860 & $9.993_{-61}^{+75}$ & 1.476 & 0.136 & 0 & 1.12 & 0.440 \\
$\Upsilon\left(1^{3} P_{1}\right)$ & 9.893 & $10.002_{-63}^{+78}$ & 1.500 & 0.122 & 0 & 1.10 & 0.448 \\
$\Upsilon\left(1^{3} P_{2}\right)$ & 9.913 & $10.011_{-65}^{+79}$ & 1.521 & 0.109 & 0 & 1.09 & 0.455 \\
$\Upsilon\left(2^{3} S_{1}\right)$ & 10.023 & $10.084_{-76}^{+93}$ & 1.593 & 0.111 & 0 & 1.03 & 0.478 \\
$\Upsilon\left(2^{3} P_{0}\right)$ & 10.232 & $*$ & $*$ & $*$ & $*$ & $*$ & $*$ \\
$\Upsilon\left(2^{3} P_{1}\right)$ & 10.255 & $*$ & $*$ & $*$ & $*$ & $*$ & $*$ \\
$\Upsilon\left(2^{3} P_{2}\right)$ & 10.268 & $*$ & $*$ & $*$ & $*$ & $*$ & $*$ \\
$\Upsilon\left(3^{3} S_{1}\right)$ & 10.355 & $*$ & $*$ & $*$ & $*$ & $*$ & $*$ \\
$\Upsilon\left(4^{3} S_{1}\right)$ & 10.580 & $*$ & $*$ & $*$ & $*$ & $*$ & $*$ \\
$B_{c}\left(1^{1} S_{0}\right)$ & $6.4 \pm 0.4$ & $6.309_{-3}^{+5}$ & 0.698 & 0.181 & 0 & 1.53 & 0.347 \\
\hline \hline
\end{tabular}


TABLE V. Variations of the theoretical predictions of Table II when the uncertainties (3) (i) and (ii) of Sec. IV C are separately taken into account. The number in bracket shows a naive estimate of uncertainties from different $\varepsilon$-expansion schemes. Those values corresponding to unreliable theoretical predictions are indicated with a star $(*)$. The input parameter is $\alpha_{\mathrm{s}}\left(M_{Z}\right)=0.1181$. The scale is fixed according to the procedure (A) of Eq. (53). All dimensionful numbers are in $\mathrm{MeV}$.

\begin{tabular}{|c|c|c|c|}
\hline & \multirow[b]{3}{*}{$\delta \bar{m}_{b}$} & \multicolumn{2}{|c|}{ Uncertainties from higher-order corrections } \\
\hline & & (i) & (ii) \\
\hline & & +2 & $\pm 3( \pm 25)$ \\
\hline \multirow{10}{*}{$\delta E_{X}$} & $Y\left(1^{3} P_{0}\right)$ & -31 & \pm 21 \\
\hline & $Y\left(1^{3} P_{1}\right)$ & -32 & \pm 22 \\
\hline & $\Upsilon\left(1^{3} P_{2}\right)$ & -33 & \pm 22 \\
\hline & $\Upsilon\left(2^{3} S_{1}\right)$ & -39 & \pm 10 \\
\hline & $\Upsilon\left(2^{3} P_{0}\right)$ & -99 & \pm 102 \\
\hline & $Y\left(2^{3} P_{1}\right)$ & -100 & \pm 116 \\
\hline & $\Upsilon\left(2^{3} P_{2}\right)$ & -101 & \pm 129 \\
\hline & $Y\left(3^{3} S_{1}\right)$ & -108 & \pm 140 \\
\hline & $\Upsilon\left(4^{3} S_{1}\right)$ & $*$ & $*$ \\
\hline & $B_{c}\left(1^{1} S_{0}\right)$ & -3 & \pm 17 \\
\hline
\end{tabular}

and to increase the $n=2$ and $n=3$ levels by about 70-100 $\mathrm{MeV}$ and 240-280 MeV, respectively.

We have analyzed the uncertainties of our predictions within perturbative QCD. The uncertainties originating from the error of the input $\alpha_{\mathrm{s}}^{(5)}\left(M_{Z}\right)$ [Sec. IV C: (2)] are as large as (in most cases larger than) other uncertainties, reflecting a high sensitivity of the bottomonium spectrum to $\alpha_{\mathrm{s}}^{(5)}\left(M_{Z}\right)$. The uncertainties from unknown higher-order corrections [Sec. IV C: (3) and (4)] are consistent with the estimates

TABLE VI. Variations of the theoretical predictions of Table II when the uncertainties (4) (i) and (ii) of Sec. IV C are separately taken into account. Those values corresponding to unreliable theoretical predictions are indicated with a star $(*)$. The input parameter is $\alpha_{\mathrm{s}}\left(M_{Z}\right)=0.1181$. All dimensionful numbers are in $\mathrm{MeV}$.

\begin{tabular}{|c|c|c|c|}
\hline & & Uncer & le fixing \\
\hline & & (i) & (ii) \\
\hline & $\delta \bar{m}_{b}$ & 0 & +1 \\
\hline & $Y\left(1^{3} P_{0}\right)$ & -2 & -13 \\
\hline & $Y\left(1^{3} P_{1}\right)$ & -2 & -13 \\
\hline & $Y\left(1^{3} P_{2}\right)$ & -1 & -14 \\
\hline & $Y\left(2^{3} S_{1}\right)$ & 0 & -23 \\
\hline$\delta E_{X}$ & $\Upsilon\left(2^{3} P_{0}\right)$ & $*$ & -112 \\
\hline & $Y\left(2^{3} P_{1}\right)$ & $*$ & -118 \\
\hline & $Y\left(2^{3} P_{2}\right)$ & $*$ & -123 \\
\hline & $Y\left(3^{3} S_{1}\right)$ & $*$ & -183 \\
\hline & $Y\left(4^{3} S_{1}\right)$ & $*$ & $*$ \\
\hline & $B_{c}\left(1{ }^{1} S_{0}\right)$ & +2 & -4 \\
\hline
\end{tabular}

TABLE VII. Summary of the uncertainties discussed in Sec. IV C. The figures are obtained as \pm the absolute value of the maximum of the corresponding uncertainties. In the column labeled "h.o. corrections" the maximum is taken from the uncertainties of Tables V and VI. All dimensionful numbers are in $\mathrm{MeV}$.

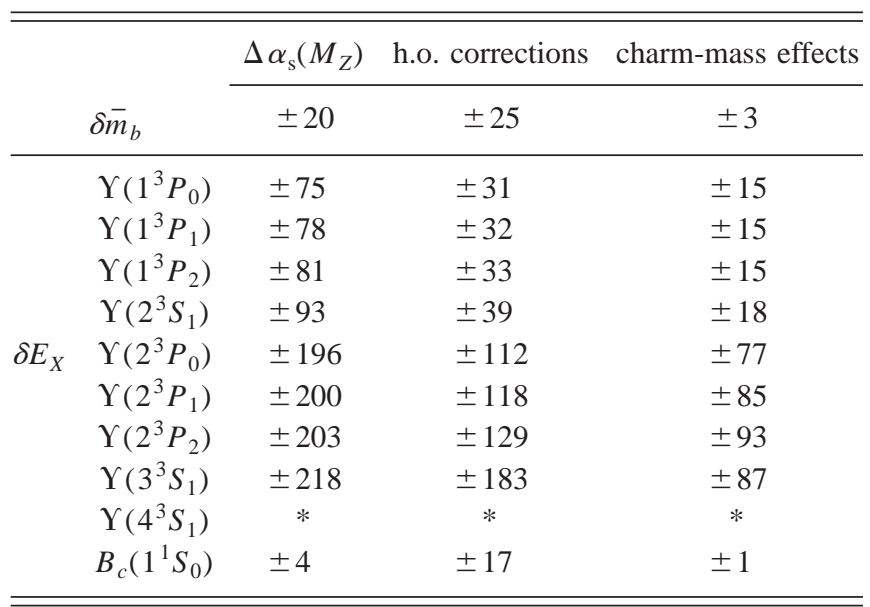

based on the next-to-leading order renormalons, i.e. are numerically of the same size as $\Lambda_{\mathrm{QCD}} \times\left(a_{X} \Lambda_{\mathrm{QCD}}\right)^{2}$ : if we approximate $1 / a_{X} \simeq \mu_{X}^{A}$, take the values of Table II, and $\Lambda_{\mathrm{QCD}}$ $=300-500 \mathrm{MeV}$, we obtain for the $1 S$ state a contribution of order $\pm(5-30) \mathrm{MeV}$, for the $n=2$ states a contribution of order $\pm(20-130) \mathrm{MeV}$ and for the $n=3$ states a contribution of order \pm (40-220) MeV. The uncertainties of the charm-mass effects have been discussed in Sec. IV C: (1).

If we take a lower value $(\simeq 0.1161)$ of the input $\alpha_{\mathrm{s}}^{(5)}\left(M_{Z}\right)$ within its present error, the theoretical predictions for the bottomonium spectrum are in agreement with the experimental data within the next-to-leading renormalon uncertainties. Considering the high sensitivity to $\alpha_{\mathrm{s}}^{(5)}\left(M_{Z}\right)$, the agreement (inside the present world-average values) seems to be quite nontrivial. Thus, the data at our disposal would confirm the analysis done in [2]: (1) the bulk of the bottomonium spectrum is accessible by perturbative QCD up to the $n=3$ states (at least in the scheme of Table II); (2) nonperturbative con-

TABLE VIII. Pure charm-mass correction of order $\varepsilon^{2}$ and $\varepsilon^{3}$ to Eq. (49) (last three lines). The input parameter is $\alpha_{\mathrm{s}}\left(M_{Z}\right)=0.1181$. All quantities are in $\mathrm{GeV}$.

\begin{tabular}{cccc}
\hline \hline State $X$ & $\mu_{X}^{A}$ & Eq. (61) & Eq. (62) \\
\hline$\Upsilon\left(1^{3} S_{1}\right)$ & 2.34 & 0.019 & 0.010 \\
$\Upsilon\left(1^{3} P_{0}\right)$ & 1.08 & 0.059 & 0.025 \\
$\Upsilon\left(1^{3} P_{1}\right)$ & 1.05 & 0.061 & 0.024 \\
$\Upsilon\left(1^{3} P_{2}\right)$ & 1.02 & 0.064 & 0.023 \\
$\Upsilon\left(2^{3} S_{1}\right)$ & 1.00 & 0.070 & 0.035 \\
$\Upsilon\left(2^{3} P_{0}\right)$ & 0.68 & 0.192 & 0.041 \\
$\Upsilon\left(2^{3} P_{1}\right)$ & 0.67 & 0.202 & 0.035 \\
$\Upsilon\left(2^{3} P_{2}\right)$ & 0.65 & 0.226 & 0.017 \\
$\Upsilon\left(3^{3} S_{1}\right)$ & 0.69 & 0.188 & 0.068 \\
$\Upsilon\left(4^{3} S_{1}\right)$ & $*$ & $*$ & $*$ \\
\hline \hline
\end{tabular}


tributions may be of the type associated with the next-toleading renormalon as estimated above.

If the true value of $\alpha_{\mathrm{s}}^{(5)}\left(M_{Z}\right)$ turns out to be close to its upper value of 0.1201 , however, independent nonperturbative effects, which cannot be absorbed into the next-toleading renormalons, should exist, with magnitudes of at least about $60 \mathrm{MeV}$ for the $n=2$ states and at least about 260 $\mathrm{MeV}$ for the $n=3$ states. In this case, the nonperturbative effects should decrease the higher levels, resulting in a tendency opposite to that of the effects usually considered by local gluon condensates or by a linear confining potential. Unless $\alpha_{\mathrm{s}}^{(5)}\left(M_{Z}\right)$ is known more precisely, we cannot constrain the nonperturbative effects more stringently with our method. On the other hand, once $\alpha_{\mathrm{s}}^{(5)}\left(M_{Z}\right)$ is measured more precisely in the future, it is certain that we will be able to investigate the nonperturbative effects on the bottomonium states very much in detail, since there are a number of energy levels that can be computed reliably in the present perturbative method.

Yet another possible approach is to take advantage of the high sensitivity of the bottomonium spectrum to $\alpha_{\mathrm{s}}^{(5)}\left(M_{Z}\right)$ and to determine both $\alpha_{\mathrm{s}}^{(5)}\left(M_{Z}\right)$ and $\bar{m}_{b}$ from a fit to the experimental data, assuming that nonperturbative effects can be absorbed into the next-to-leading renormalon uncertainties. Then we may compare the value of $\alpha_{\mathrm{s}}^{(5)}\left(M_{Z}\right)$ with those obtained from other measurements, for a consistency check of the assumption. It is a meaningful method to study the nature of the nonperturbative effects.

Therefore, according to our observation that the perturbative predictions, within uncertainties, are consistent with the experimental data, we present the value of the bottom quark $\overline{\mathrm{MS}}$ mass as determined from the $\mathrm{Y}(1 S)$ mass in our approach

$$
\bar{m}_{b}=4190 \pm 20 \pm 25 \pm 3 \mathrm{MeV} \text {, }
$$

where the first error refers to the uncertainty in $\alpha_{\mathrm{s}}^{(5)}\left(M_{Z}\right)$, the second error to the higher-order corrections, and the third error to the uncertainty of the charm-mass corrections. It is useful to compare the above result with a recent estimate done in [28], $\bar{m}_{b}=4210 \pm 25 \pm 90$, where the renormalon cancellation has been implemented in a scheme different from the $\varepsilon$ scheme adopted here. Taking into account that in [28] charm-mass effects have not been included in the calculation (only added to the errors) the central value of $4210 \mathrm{MeV}$ is consistent, within errors, with our central value of $4190 \mathrm{MeV}$ [or, with the value of Eq. (29)]. The error $\pm 25 \mathrm{MeV}$ refers to uncertainties in $\alpha_{\mathrm{s}}^{(5)}\left(M_{Z}\right)$ and corresponds to our $\pm 20 \mathrm{MeV}$. The error $\pm 90 \mathrm{MeV}$ refers to charm-mass effects, higherorder perturbative and nonperturbative corrections. It corresponds to our errors $\pm 25 \pm 3 \mathrm{MeV}$. Subtracting from $\pm 90 \mathrm{MeV}$ a $\pm(10-20) \mathrm{MeV}$ estimate of the charm-mass effects that have not been calculated explicitly in [28], but have been included in our analysis, it remains a $\pm(40-$ 50) $\mathrm{MeV}$ difference between the two error estimates. The difference is given by the size of the nonperturbative correc- tions. These are guessed to be $\pm 50 \mathrm{MeV}$ in [28]. On the other hand, in the present work, the observation that the (perturbative) determinations of the $n=2$ and $n=3$ bottomonium levels agree within the present uncertainties with the experimental data suggests this to be also the case for the $n=1$ level, i.e. for $\bar{m}_{b}$. In other words, nonperturbative effects would be absorbed into the present uncertainties. Hence, higher bottomonium levels provide the additional physical input needed in order to constrain the nonperturbative corrections on the $b$ mass. We also mention that in the analysis of [7], where $\bar{m}_{b}$ has been extracted from the $Y$ sum rules including charm-mass effects, the author obtains $\bar{m}_{b}$ $=4.17 \pm 0.05 \mathrm{GeV}$. This figure is consistent with our estimate.

Let us, finally, remark some general tendencies of the perturbative predictions. First, evolving $\alpha_{\mathrm{s}}^{\left(n_{l}\right)}(\mu)$ from $\alpha_{\mathrm{s}}^{(5)}\left(M_{Z}\right)$ using the 3-loop running instead of the 4-loop running, is theoretically still consistent within our present accuracy. In this case, the level spacings of the spectrum become smaller, since the 4-loop coefficient of the beta function is negative. Secondly, if we compare the analytic runningcoupling constant (defined in Sec. II B and used throughout this paper) and the numerical running coupling constant (used in [2]), the former is larger at relevant scales. Hence, the level spacings become smaller if we use the latter coupling. These variations of the predictions are certainly higher-order uncertainties. Nevertheless, it is surely worthwhile to further examine the quality of the theoretical predictions carefully and to seek for most reliable theoretical predictions at the current reachable accuracy.

\section{ACKNOWLEDGMENTS}

Y.S. was supported in part by the Japan-Germany Cooperative Science Promotion Program. The work of A.V. has been supported by the European Community through the Contract No. HPMF-CT-2000-00733. A.V. acknowledges discussions with Antonio Pineda.

\section{APPENDIX A: POLE MASS EXPANSION COEFFICIENTS}

Here, we give the analytic expressions for the coefficients $d_{1}^{\left(n_{l}\right)}$ and $d_{2}^{\left(n_{l}\right)}$ that appear in Eq. (21). The coefficient $d_{1}^{\left(n_{l}\right)}$ has been calculated in [6], while the analytic expression of $d_{2}^{\left(n_{l}\right)}$ can be derived from the result of $[30],{ }^{11}$ the renormalization-group evolutions of $\alpha_{\mathrm{s}}^{\left(n_{l}\right)}(\mu)$ and $m_{\overline{\mathrm{MS}}}(\mu)$, and the matching condition $[31]:^{12}$

\footnotetext{
${ }^{11}$ This constant was obtained numerically in [29] in a certain approximation.

${ }^{12}$ This relation coincides with Eq. (14) of [30], which is given numerically (indirectly through $\beta_{0}$ and $\beta_{1}$ ). Note that, in the other formulas of [30], the coupling of the full theory is used.
} 


$$
\begin{aligned}
d_{1}^{\left(n_{l}\right)}= & \frac{307}{32}+\frac{\pi^{2}}{3}+\frac{\pi^{2} \ln 2}{9}-\frac{\zeta_{3}}{6}+n_{l}\left(-\frac{71}{144}-\frac{\pi^{2}}{18}\right) \\
\simeq & 13.4434-1.04137 n_{l}, \\
d_{2}^{\left(n_{l}\right)}= & \frac{8462917}{93312}+\frac{652841 \pi^{2}}{38880}-\frac{695 \pi^{4}}{7776}-\frac{575 \pi^{2} \ln 2}{162}-\frac{22 \pi^{2} \ln ^{2} 2}{81}-\frac{55 \ln ^{4} 2}{162}-\frac{220 \mathrm{Li}_{4}\left(\frac{1}{2}\right)}{27}+\frac{58 \zeta_{3}}{27}-\frac{1439 \pi^{2} \zeta_{3}}{432} \\
& +\frac{1975 \zeta_{5}}{216}+n_{l}\left(-\frac{231847}{23328}-\frac{991 \pi^{2}}{648}+\frac{61 \pi^{4}}{1944}-\frac{11 \pi^{2} \ln 2}{81}+\frac{2 \pi^{2} \ln ^{2} 2}{81}+\frac{\ln ^{4} 2}{81}+\frac{8 \operatorname{Li}_{4}\left(\frac{1}{2}\right)}{27}-\frac{241 \zeta_{3}}{72}\right) \\
& +n_{l}^{2}\left(\frac{2353}{23328}+\frac{13 \pi^{2}}{324}+\frac{7 \zeta_{3}}{54}\right) \\
\simeq & 190.391-26.6551 n_{l}+0.652691 n_{l}^{2} .
\end{aligned}
$$

\section{APPENDIX B: FINITE CHARM-MASS EFFECTS IN THE ENERGY EXPANSION}

In this appendix we give the expression of $\left(\delta E_{b}\right)_{m_{c} \rightarrow \infty}^{(2)}\left(m_{b}, \rho\right)$ for a $b \bar{b}$ state of quantum numbers $n, l$. From Eq. (45) we get after explicit calculation [we write here explicitly the mass arguments, which are the $b$-pole mass and $\rho$ $\left.=2 n m_{c, \text { pole }} /\left(m_{b, \text { pole }} C_{F} \alpha_{\mathrm{s}}^{(4)}(\mu)\right)\right]$ :

$$
\begin{aligned}
& \left(\delta E_{b \bar{b}}\right)_{m_{c} \rightarrow \infty}=\varepsilon^{2}\left(\delta E_{b \bar{b}}\right)_{m_{c} \rightarrow \infty}^{(1)}\left(m_{b, \text { pole }}, \rho\right)+\varepsilon^{3}\left(\delta E_{b \bar{b}}\right)_{m_{c} \rightarrow \infty}^{(2)}\left(m_{b, \text { pole }}, \rho\right), \\
& \left(\delta E_{b \bar{b}}\right)_{m_{c} \rightarrow \infty}^{(1)}\left(m_{b, \text { pole }}, \rho\right)=\frac{m_{b, \text { pole }}\left(C_{F} \alpha_{\mathrm{s}}^{(4)}(\mu)\right)^{2}}{4 n^{2}} \frac{\alpha_{\mathrm{s}}^{(4)}(\mu)}{\pi}\left\{\frac{2}{3} \ln \left(\frac{2}{\rho}\right)-\frac{5}{9}-\frac{2}{3}\left(\psi(n+l+1)+\gamma_{E}\right)\right\}, \\
& \left(\delta E_{b \bar{b}}\right)_{m_{c} \rightarrow \infty}^{(2)}\left(m_{b, \text { pole }}, \rho\right)=\frac{m_{b, \text { pole }}\left(C_{F} \alpha_{\mathrm{s}}^{(4)}(\mu)\right)^{2}}{4 n^{2}}\left(\frac{\alpha_{\mathrm{s}}^{(4)}(\mu)}{\pi}\right)^{2}\left\{2 \beta _ { 0 } ^ { ( 4 ) } \left[-\frac{1}{2} \ln \left(\frac{2}{\rho}\right)\left(\ln \left(\frac{2}{\rho}\right)+\ln \left(\frac{m_{c, \text { pole }}}{\mu}\right)\right)+\left(\frac{1}{2} \ln \left(\frac{m_{c, \text { pole }}}{\mu}\right)\right.\right.\right. \\
& \left.+\ln \left(\frac{2}{\rho}\right)\right)\left(\psi(n+l+1)+\gamma_{E}+\frac{1}{2}\right)-\frac{2}{3} \theta(-2+n-l) \frac{\Gamma(n-l)}{\Gamma(n+l+1)} \sum_{k=0}^{n-l-2} \frac{\Gamma(2 l+2+k)}{k !(n-l-k-1)^{2}} \\
& -\frac{1}{6} \psi(1+l+n)(\psi(1+l+n)-2)-\frac{1}{3} \frac{(n-l-1) !}{(n+l) !} \sum_{k=0}^{n-l-2} \frac{(k+2 l+1) !}{k !(k+l+1-n)^{3}} \\
& -\frac{n}{3} \frac{(n+l) !}{(n-l-1) !} \sum_{k=n-l}^{\infty} \frac{k !}{(k+2 l+1) !(k+l+1-n)^{3}}-\frac{\pi^{2}}{36}-\frac{1}{2} \gamma_{E}^{2}-\frac{1}{2} \gamma_{E}-\frac{5}{72}-\frac{1}{3} \psi^{\prime}(n+l+1) \\
& \left.-\frac{1}{3}(\psi(n+l+1))^{2}-\psi(n+l+1)\left(\frac{5}{6}+\gamma_{E}\right)\right]-\frac{1}{3} \ln ^{2}\left(\frac{2}{\rho}\right) \\
& +\ln \left(\frac{2}{\rho}\right)\left(-\frac{1}{2}+\frac{2}{3} \psi(n+l+1)+\frac{2}{3} \gamma_{E}\right)-\frac{4}{9} \theta(-2+n-l) \frac{\Gamma(n-l)}{\Gamma(n+l+1)} \sum_{k=0}^{n-l-2} \frac{\Gamma(2 l+2+k)}{k !(n-l-k-1)^{2}} \\
& -\frac{1}{9} \psi(1+l+n)(\psi(1+l+n)-2)-\frac{2}{9} \frac{(n-l-1) !}{(n+l) !} \sum_{k=0}^{n-l-2} \frac{(k+2 l+1) !}{k !(k+l+1-n)^{3}} \\
& -\frac{2 n}{9} \frac{(n+l) !}{(n-l-1) !} \sum_{k=n-l}^{\infty} \frac{k !}{(k+2 l+1) !(k+l+1-n)^{3}}-\frac{\pi^{2}}{54}-\frac{13}{6} \zeta_{3}-\frac{1}{3} \gamma_{E}^{2}+\frac{1}{2} \gamma_{E}-\frac{183}{648} \\
& \left.-\frac{2}{9} \psi^{\prime}(n+l+1)-\frac{2}{9}(\psi(n+l+1))^{2}+\psi(n+l+1)\left(\frac{5}{18}-\frac{2}{3} \gamma_{E}\right)\right\} .
\end{aligned}
$$

Equation (B2) corresponds to Eq. (39); Eq. (B3) has been checked to coincide in the $m_{c} \rightarrow \infty$ limit with Eq. (64) of [7]. 
[1] W. Lucha, F. Schöberl, and D. Gromes, Phys. Rep. 200, 127 (1991); N. Brambilla and A. Vairo, in Strong Interactions at Low and Intermediate Energies, edited by J. L. Goity (World Scientific, Singapore, 2000), p. 151, hep-ph/9904330; G. Bali, Phys. Rep. 343, 1 (2001).

[2] N. Brambilla, Y. Sumino, and A. Vairo, Phys. Lett. B 513, 381 (2001).

[3] A. Hoang, M. Smith, T. Stelzer, and S. Willenbrock, Phys. Rev. D 59, 114014 (1999).

[4] M. Beneke, Phys. Lett. B 434, 115 (1998).

[5] A. Hoang, Z. Ligeti, and A. Manohar, Phys. Rev. Lett. 82, 277 (1999); Phys. Rev. D 59, 074017 (1999).

[6] N. Gray, D.J. Broadhurst, W. Grafe, and K. Schilcher, Z. Phys. C 48, 673 (1990).

[7] A. Hoang, hep-ph/0008102.

[8] D. Eiras and J. Soto, Phys. Lett. B 491, 101 (2000).

[9] N. Brambilla, A. Pineda, J. Soto, and A. Vairo, Nucl. Phys. B566, 275 (1999).

[10] A. Pineda and J. Soto, Nucl. Phys. B (Proc. Suppl.) 64, 428 (1998).

[11] S. Titard and F. Yndurain, Phys. Rev. D 49, 6007 (1994); 51, 6348 (1995).

[12] A. Pineda and F. Yndurain, Phys. Rev. D 58, 094022 (1998); 61, 077505 (2000).

[13] N. Brambilla, A. Pineda, J. Soto, and A. Vairo, Phys. Rev. D 60, 091502 (1999); Phys. Lett. B 470, 215 (1999); B. Kniehl and A. Penin, Nucl. Phys. B563, 200 (1999); B577, 197 (2000)
[14] Y. Schröder, Phys. Lett. B 447, 321 (1999); M. Peter, Phys. Rev. Lett. 78, 602 (1997).

[15] K. Melnikov and A. Yelkhovsky, Phys. Rev. D 59, 114009 (1999).

[16] N. Brambilla and A. Vairo, Phys. Rev. D 62, 094019 (2000).

[17] S.S. Gershtein, V.V. Kiselev, A.K. Likhoded, and A.V. Tkabladze, Phys. Rev. D 51, 3613 (1995).

[18] G. Martinelli and C.T. Sachrajda, Nucl. Phys. B559, 429 (1999).

[19] Y. Sumino, hep-ph/0004087.

[20] D.E. Groom et al., Eur. Phys. J. C 15, 1 (2000).

[21] K. Chetyrkin, B. Kniehl, and M. Steinhauser, Phys. Rev. Lett. 79, 2184 (1997).

[22] M. Melles, Phys. Rev. D 62, 074019 (2000).

[23] M. Melles, Nucl. Phys. B (Proc. Suppl.) 96, 472 (2001).

[24] Y. Sumino, hep-ph/0104259.

[25] A.A. Penin and A.A. Pivovarov, Nucl. Phys. B549, 217 (1999).

[26] A. Hoang, Phys. Rev. D 61, 034005 (2000); M. Beneke and A. Signer, Phys. Lett. B 471, 233 (1999).

[27] M. Eidemüller and M. Jamin, Phys. Lett. B 498, 203 (2001).

[28] A. Pineda, J. High Energy Phys. 06, 022 (2001).

[29] K. Chetyrkin and M. Steinhauser, Phys. Rev. Lett. 83, 4001 (1999); Nucl. Phys. B573, 617 (2000).

[30] K. Melnikov and T. van Ritbergen, Phys. Lett. B 482, 99 (2000).

[31] S. Larin, T. van Ritbergen, and J. Vermaseren, Nucl. Phys. B438, 278 (1995). 